\title{
دور الفكر الجيوبوليتيكي في دعم إسرائيل
}

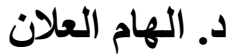
أستاذ مساعد، كلية الاقتصاد والإدارة ـ قسم العلوم السبياسية

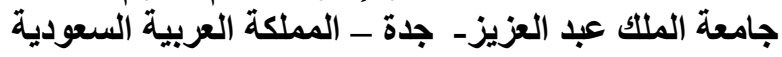
البريد الاككتروني: gmail.com

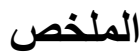

تهذف الدراسة إلى وضع تصور واضح حول مصطلح الجيوبوليتيك، والتطرق لتوجه إسرائيل نحو التوسع الإقليمي ودعم وجودها الحيوي في خارطة العالم، بالإضافة لإعطاء مؤشرات عن مستقبل منطقة الثرق الأوسط في ضوء سعيها للتوسع الاقتصادي والسياسي ، وتضمنت الفرضية إجابات نموذجية حول مشكلة الدراسة،

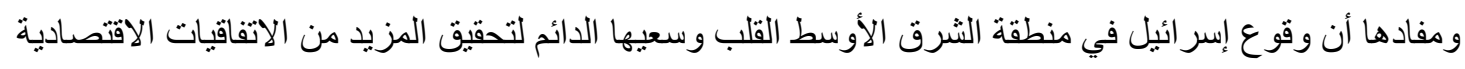

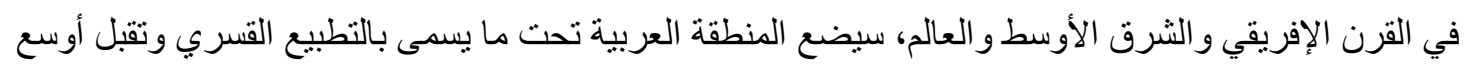
للوجود الإسر ائيلي. ولتحقيق أهداف الدراسة والإجابة عن اسئلتها تم اعتماد المنهج التحليلي و المنهج الموروفولوجي بالإضافة للمنهج

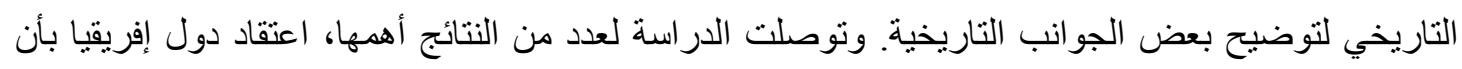
بناء علاقات فاعلة مع إسرائيل سيؤدي إلى تعزيز علاقتها مع الولايات المتحدة الأمريكية، والعلاقة الصينية الإسرائيلية مبنية على فصل الملفات السياسية عن الاتفاقيات الاقتصادية بين البلاين، والعلاقات الهندية الإسر ائيلية تقوم على تطويق إسرائيل لأكبر قوة وحيدة في العالم الإسلامي وهي باكستان، وفي المقابل تفوز

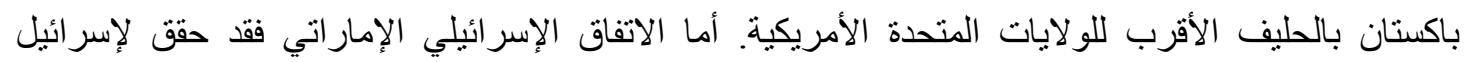

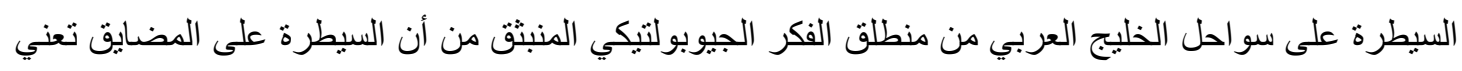

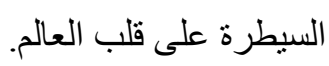

الكلمات المفتاحية: الجيوبوليتيك، إسر ائيل. 


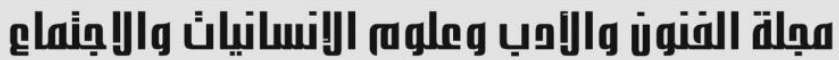 \\ Journal of Arts, Literature, Humanities and Social Sciences www.jalhss.com Volume (63) January 2021 \\ العدد (63) يناير 2021 (الع \\ The Role of Geopolitical Thought in Support of Israel
}

\author{
Dr. Elham Al- Allan
}

Assistant Professor, Faculty of Economics and Administration - Department of Political Science- King Abdulaziz University - Jeddah - Kingdom of Saudi Arabia Email: mh2432004@gmail.com

\begin{abstract}
The aim of this research is to identify the role of geopolitical thought in supporting Israel, and to achieve this, the geopolitical concept, the current situation and program strategy, initiatives and timetable, pivotal initiatives and enablers were revealed, and the research tool consisted in analyzing these contents and linking them to the concept of national belonging.

The descriptive method was adopted as a scientific method to address the research. The analysis indicated that the Quality of Life Program 2020 document contributes to establishing basic rules for the quality of life in the Kingdom of Saudi Arabia, which enhances the family affiliation that stems from belonging to the motherland, which indicates that satisfying the need for a citizen's community affiliation has a positive effect on deepening the sense of belonging to the homeland. He has, and this confirms the positive impact of the state in promoting belonging to the homeland by activating its various functions.
\end{abstract}

Keywords: Geopolitical Thought, Israel. 


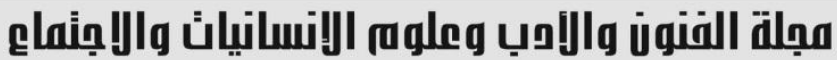

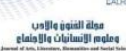

Journal of Arts, Literature, Humanities and Social Sciences

www.jalhss.com

Volume (63) January 2021

العدد (63) يناير 2021

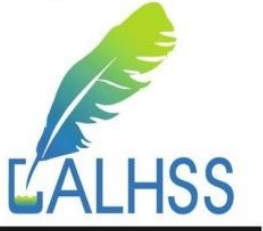

المقدمة

استطاعت إسرائيل منذ احتلالها للأراضي الفلسطينية عام 1948م، من ترسيخ وجودها عبر كافة الوسائل

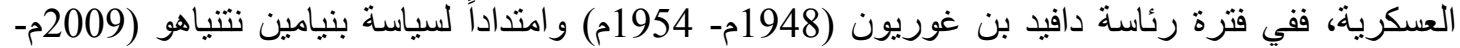

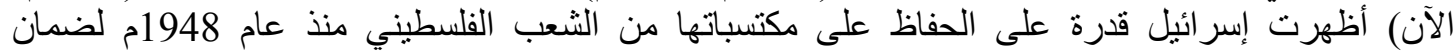

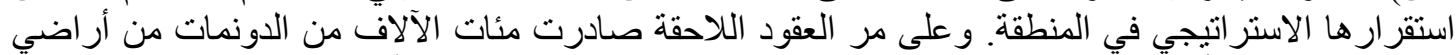

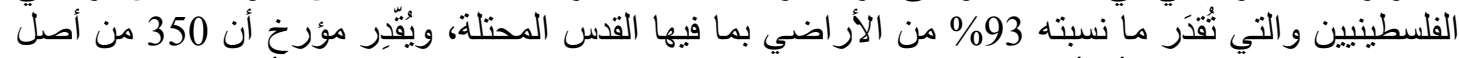

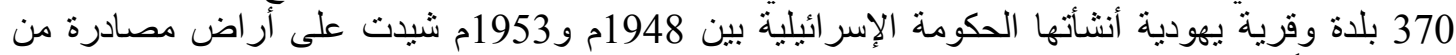

فلسطينيين.

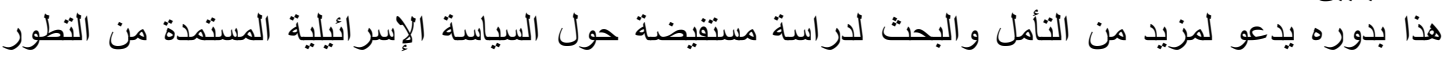

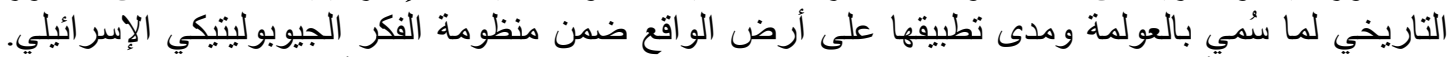

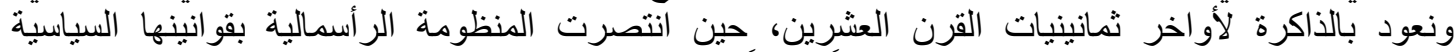

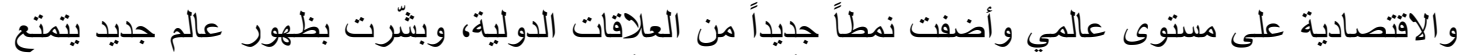

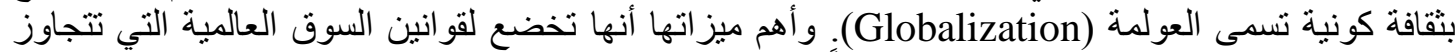

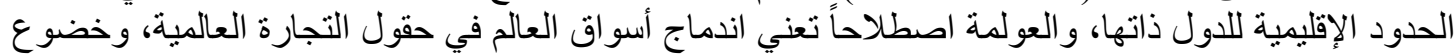

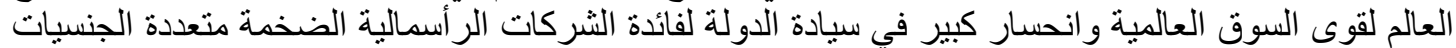

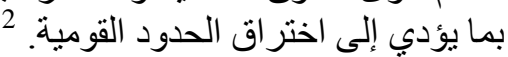

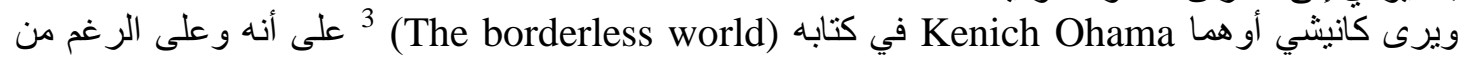
وجود الحدود الطبيعية بين الدول، لكنه في عالم السياسة وخريطة التنافس الاقتصادي والصناعي هذه الحدود مضمحلة وزائلة.

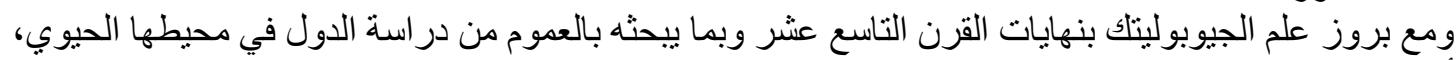

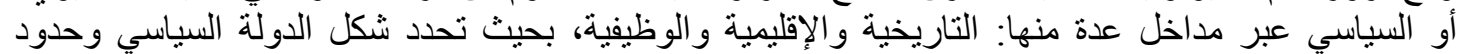

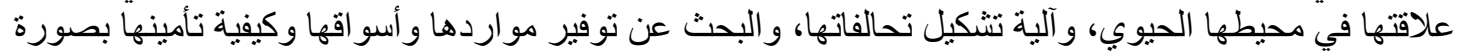

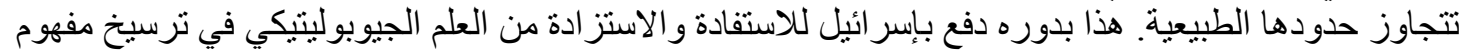

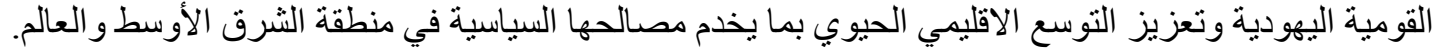

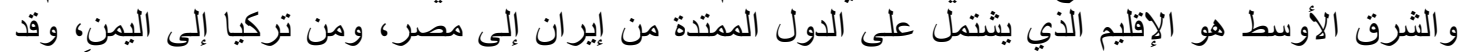

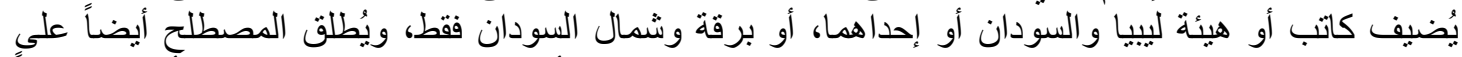

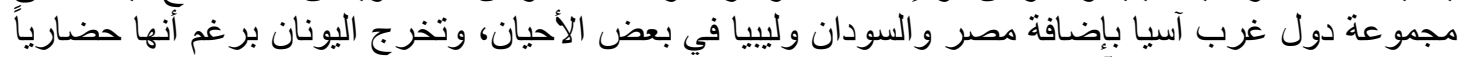

تشبه إقليم الثرق الأوسط.

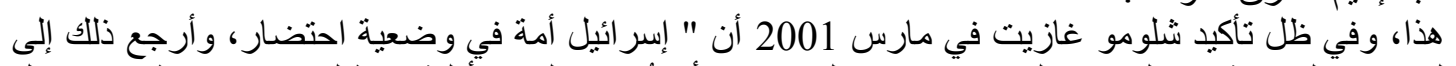

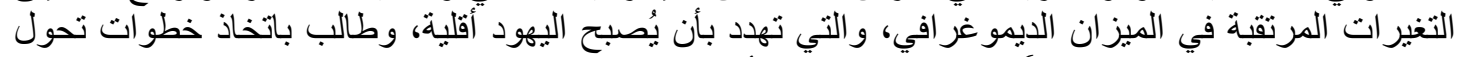

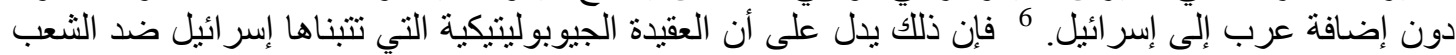

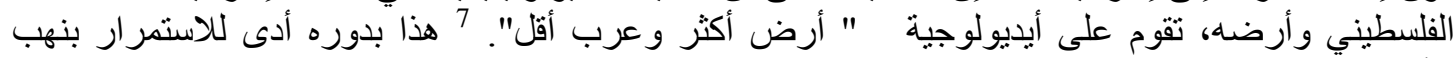

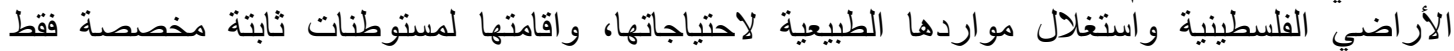
للإسر ائيليين وضم مناطق من الضفة الغربية لنفوذها خلافاً للقانون الدولئهاني.

1- منظمة حقوق الإنسان، إسر ائيل: سياسات الأراضي التمييزية تحصر الفلسطينيين عبر الرابط الالكتروني: https://www.hrw.org/ar/news/2020/05/12/339933

2- سفين جلال فتح الله، دراسات جيو بوليتيكية، مركز كردستان للارراسات الاستر اتيجية، 2013، ص31 http:// www.amazon.com/ dp/0887309674-3

Kenichi Ohama, "Managing in borderless world" , Harvard business review,5-6/1989 -4 5- محمد رياض، الأصول العامة في الجغر افيا السياسية والجيوبوليتيك، (2012)، مؤسسة هنداوي للتعليم و الثقافة،

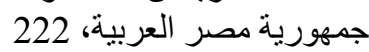
6- غازيت شلومو، 2005، خيار إسر ائيل ما بعد فلك الارتباط، في: ما بعد فلك الارتباط: سيناريو هات إسر ائيلية، سلسلة

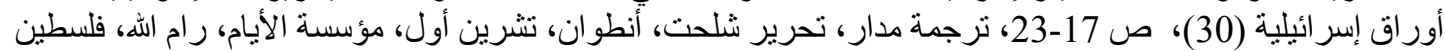

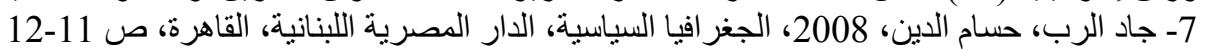
8- مركز المعلومات الإسرائيلي لحقوق الإنسان في الأراضي المحتلة (بنسليم)، إنهاء الاحتلال، 2017، الإنة عبر الرابط

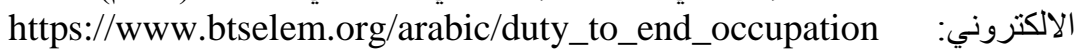




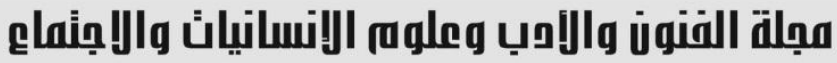

Journal of Arts, Literature, Humanities and Social Sciences

www.jalhss.com

Volume (63) January 2021

العدد (63) يناير 2021

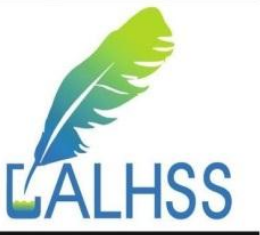

إثكالية الدراسة

بات الصر اع الفلسطيني الإسر ائيلي عصي على الحل، و التوصل لقر ار جذري بإلغاء الاحتلال بات شُبه مستحيل،

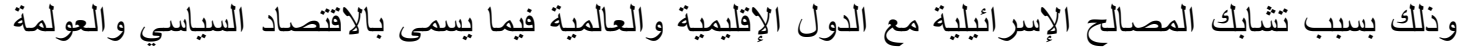

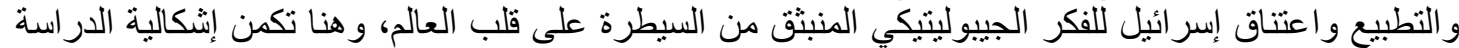

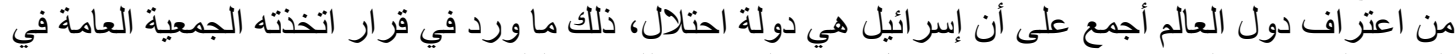

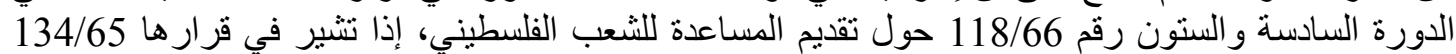

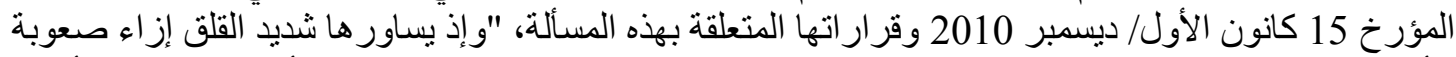

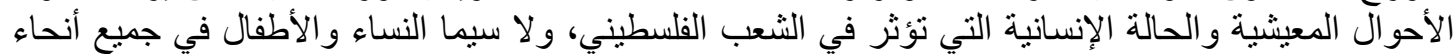

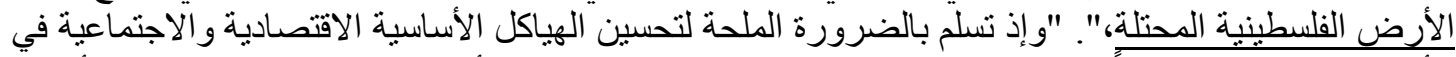

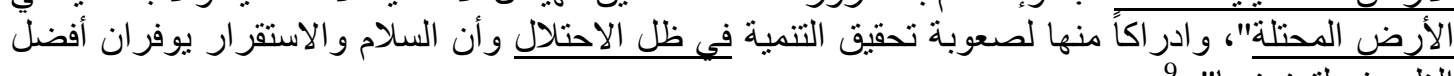
الظروف لتعزيز ها". ومع ذلك يتم عقد الاتفاقيات (كينيا، اثيو بيا، الصين،)، التحالفات (الولايات المتحدة الأمريكية)، و عمليات السلام

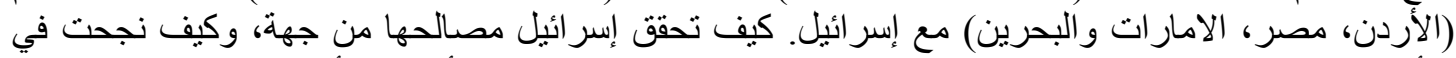

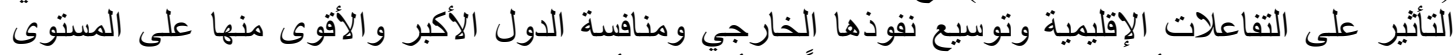

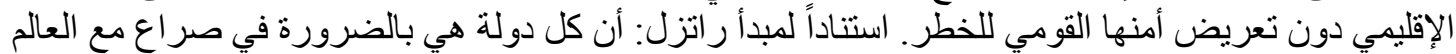

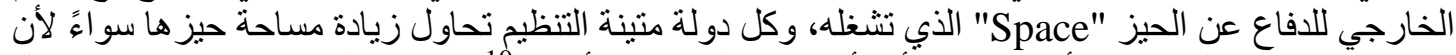

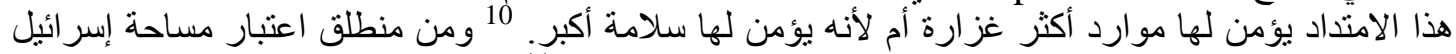

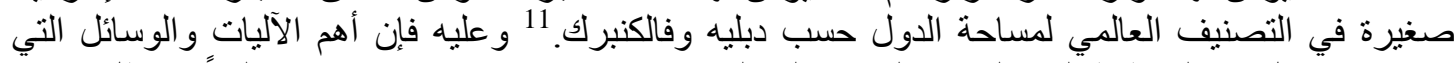

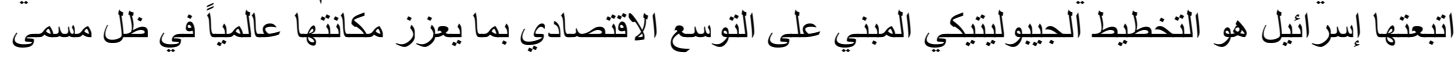

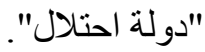

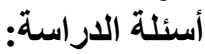
هناك مجمو عة من الأسئلة تحاول هذه الدر اسة تقديم أجوبة عليها فيما يتعلق بالجيوبوليتيك، وتشمل: 1- ما هو موقع إسر ائيل في الخارطة السياسية؛ 2- كيف حددت نظريات الجيوبوليتيك موقع إسر ائيل في خريطة العالم السياسي؟

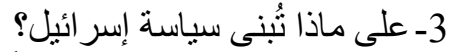

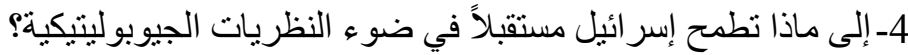
فرضية الدراسة:

تتضمن الفرضية إجابات نموذجية على مشكلة الإسلة الدراسة:

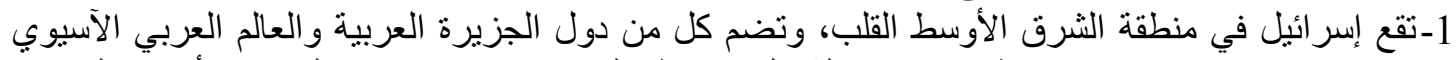

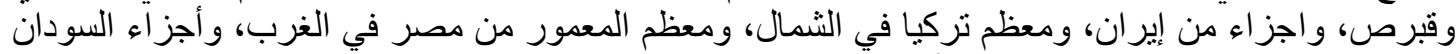
و واثيو بيا والصومال واجز المطلة على البحر الأحمر وخليج عدن.

2- سعت إسر ائيل لدعم اقتصادها السياسي بالعديد من الاتفاقيات في الثرق الأليات الأوسط و القرن الإفريقي و العالم.

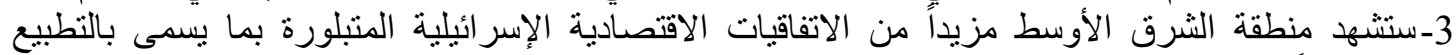

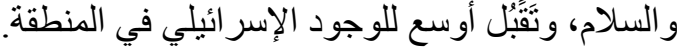

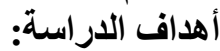

1-وضع تصور و اضح حول مصطلح الجيوبوليتيك.

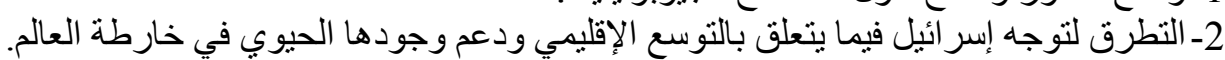

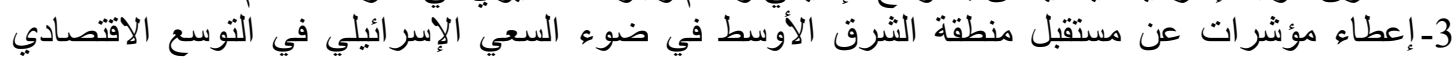
الحدود المكانية والزمانية للاراسة:

9-18_a

https://www.unescwa.org/sites/www.unescwa.org/files/un_resolutions/a_res_66_118_a.pdf 10- محمد أزهر سعيد السماك، الجغر افيا السياسية- أسس وتطبيقات، (1988)، مديرية دار الكتب للطباعة والنشر، 


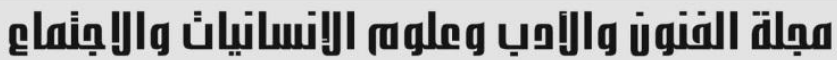

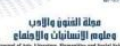

Journal of Arts, Literature, Humanities and Social Sciences

www.jalhss.com

Volume (63) January 2021

العدد (63) يناير 2021

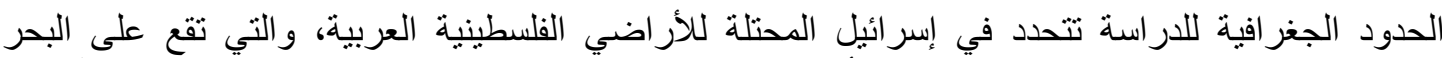

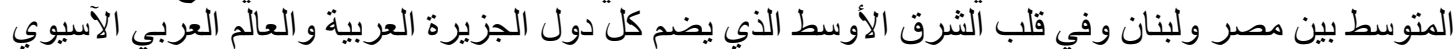

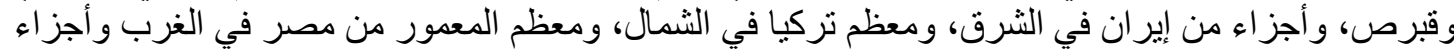
السودان واثيوبيا والصومال إيران المطلة على البحر الأحمر وخليج عدن. موقعها المهم وسياستها الجيوبوليتيكية الئية

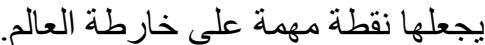

أما الدود الفلكية للمنطقة فإنها تقع بين دائرتي عرض (30-31) درجة شمالاً، و(45-34) درجة شرقاً.

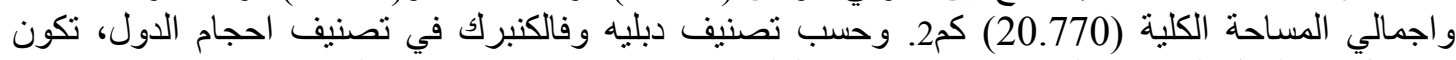

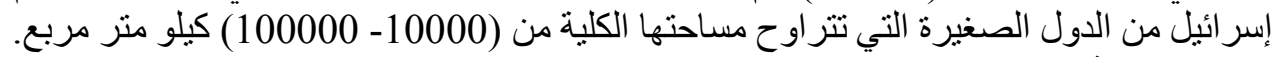

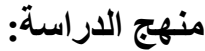

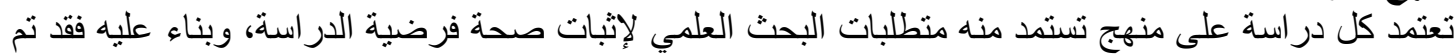

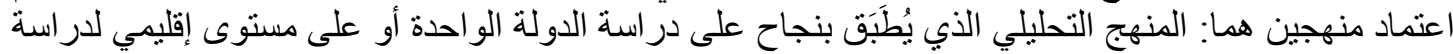

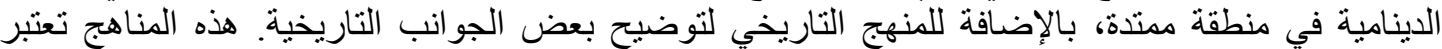
ظاهرة الجغر افيا السياسية بحاجة لعدة جو انب وليس الإلمام بجانب و احد فقط وذلك لتحفي لتحقيق أهداف الدراسة.

مفهوم الجيوبوليتيك

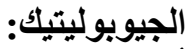

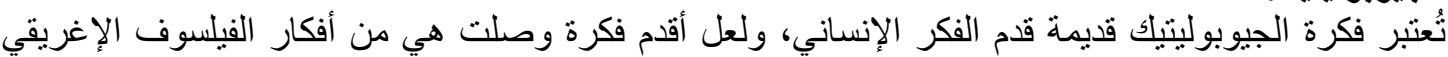

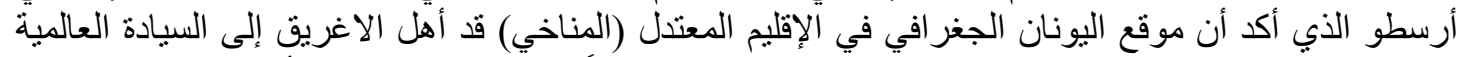

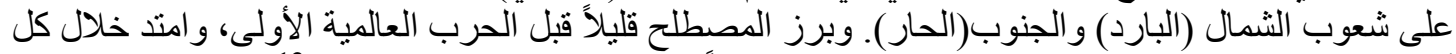

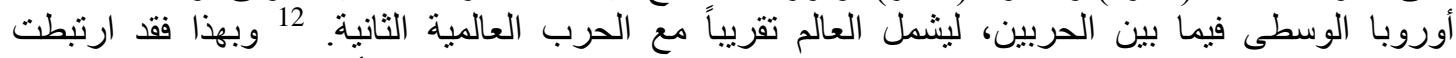

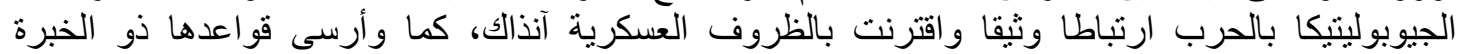

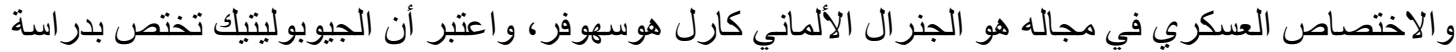

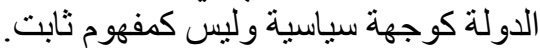

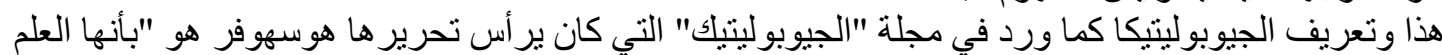

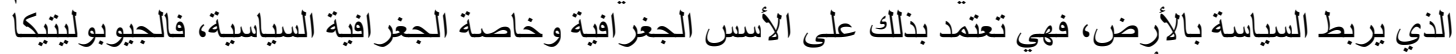

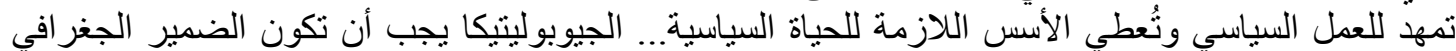
للاولة". 13

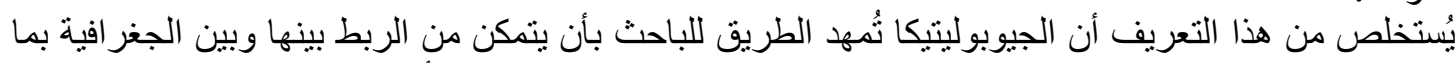

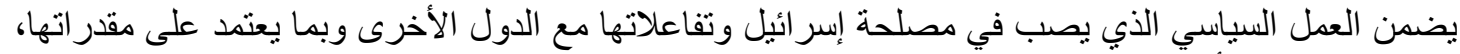

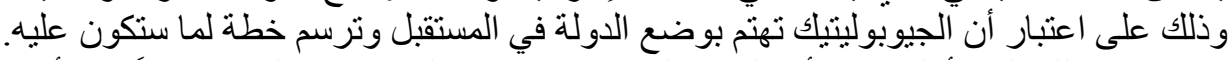

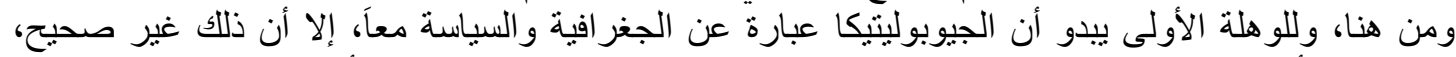

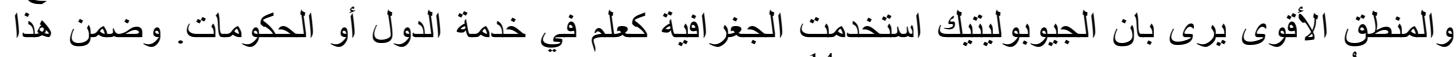

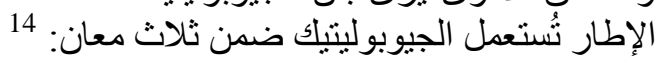

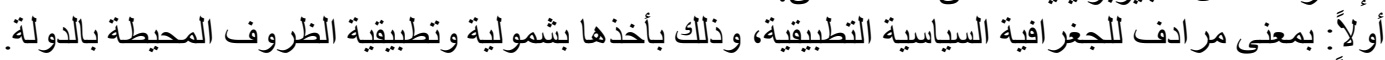

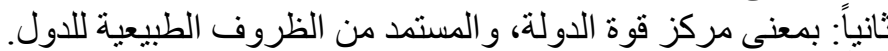

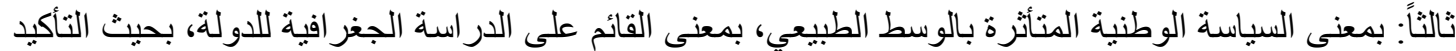

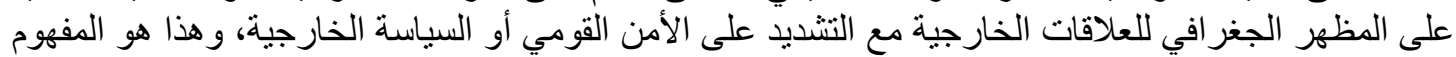

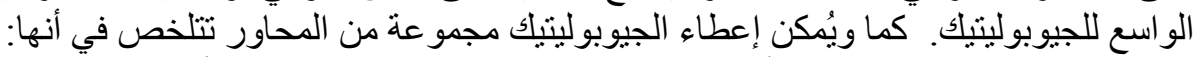

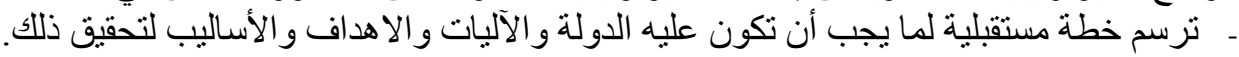
تعمل على جعل حقائق الجغر افية مكرسة لخدمة الدول بل بما فيها البحار و الحدود و الموقع ...الخ.

12- عاطف علبي، الجغر افيا الاقتصادية والسياسية والسكانية والجيوبوليتيك، (1989)، المؤسسة الجامعية للاراسات

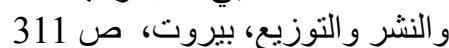

13 -Preston, J. Clarence, eds American Geography Inventory and Prospects, Syracuse 1954, p.172.

$$
\text { 14- عاطف علبي، مرجع سابق، ص } 316
$$




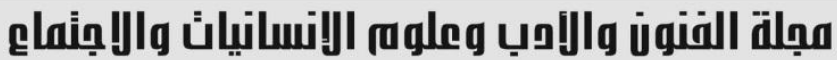

Journal of Arts, Literature, Humanities and Social Sciences www.jalhss.com

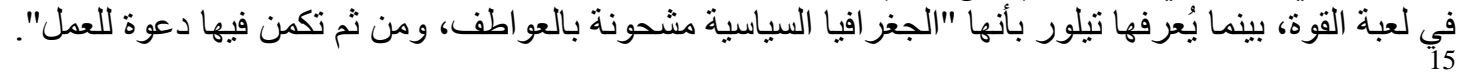

هذا يقود للتطرق لمفهوم القوة بما يخدم الدر اسة، حيث يُعرفها ديليه بأنها: "التتظيم، ويعني به الكيفية أو الطريقة

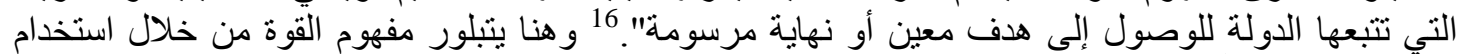

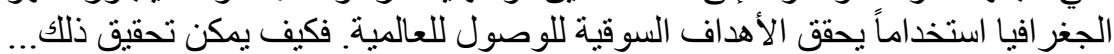

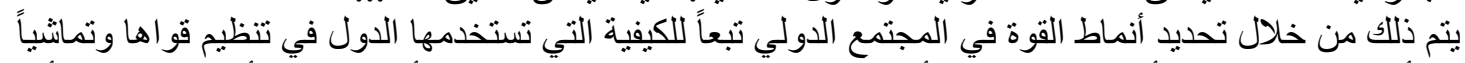

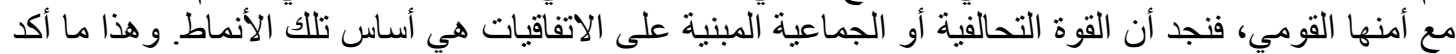

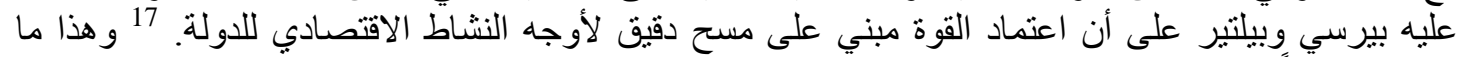

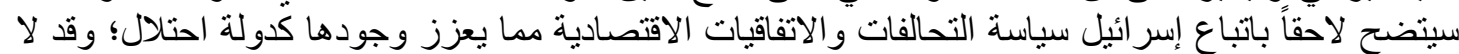

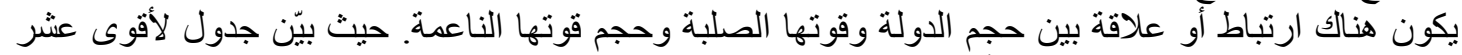

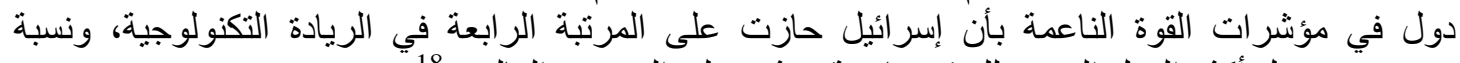

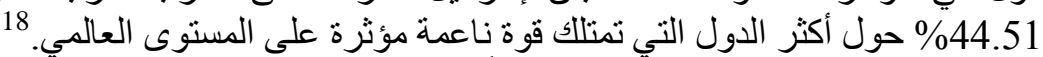

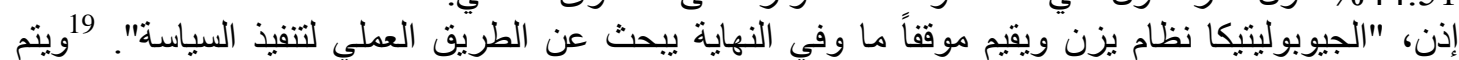

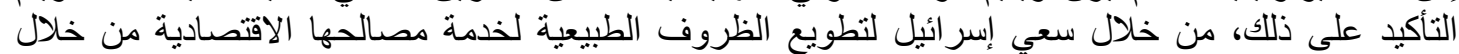

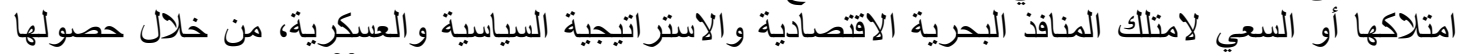

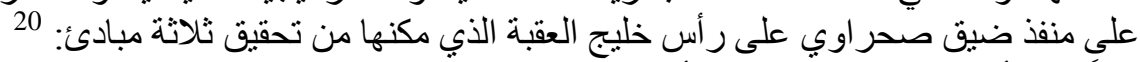

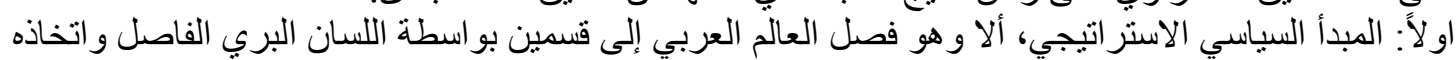

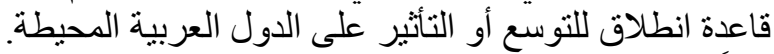

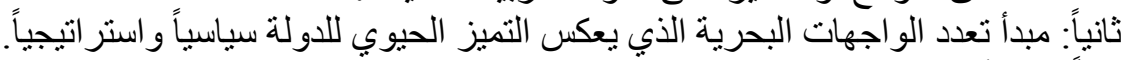

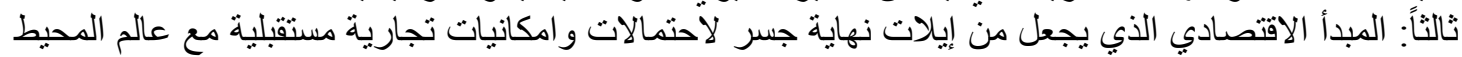

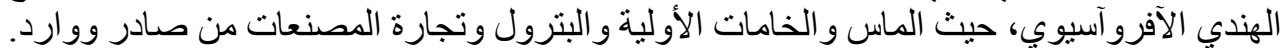

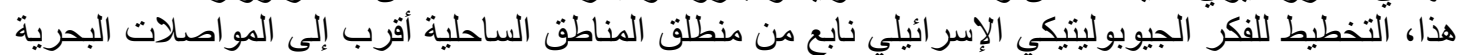

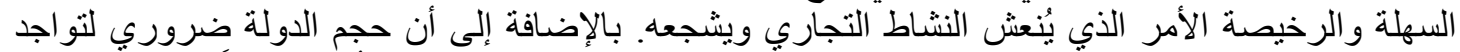

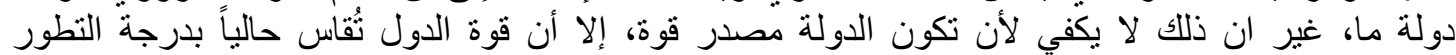

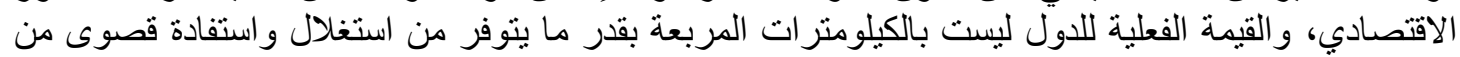

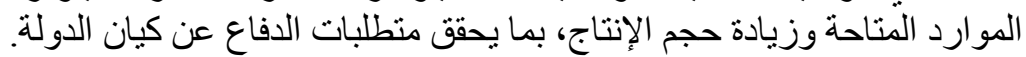

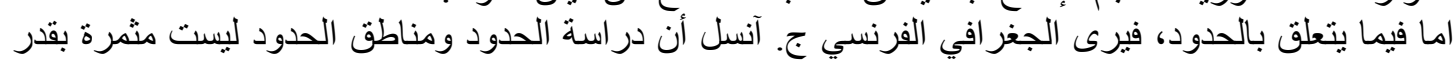

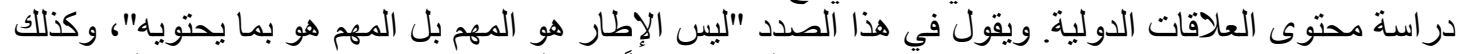

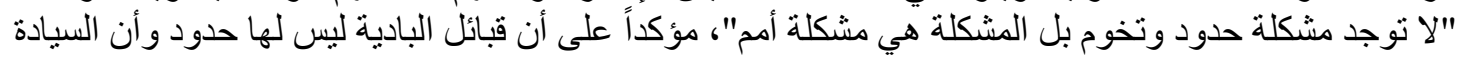

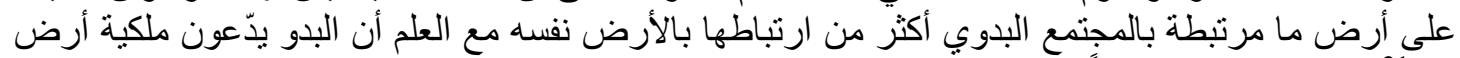
ما. 21 و هذا ما سيتضح لاحقاً خلال الدربة السة الحالية.

G.R. Taylor, Geography of an Air Age, Institute of International Affairs, London, -15

1954,p.37

16- عبد الرزاق عباس، الجغر افية السياسية مع التركيز على المفاهيم الجيوبوليتيكية، (1977)، بغداد، ص 237

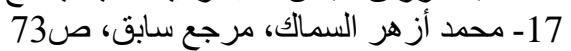

18 -McClory,J.2015,The Soft Power 30: Global Ranking of Soft Power. Portland Communications [online] Available at: www.portland- communication.com/downloads/thesoft-power-3020\% Nov.pdf [Accesses Dec30.2015]

19 -N. Pounds, Political Geography, McGraw Hill 1963, p.407

20- محمد رياض، مرجع سابق، ص246

21 -Ancel,Les Frontieres, Etudc de Geographic Politique, Recueil de Couurs, pp. 207297.1936

$$
\text { أنظر أيضاً: محمد رياض، الأصول العامة في الجغر افيا السياسية مرجع سابق، ص199 }
$$




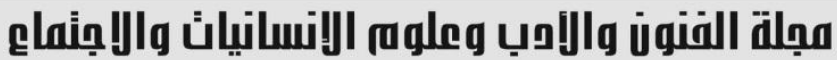

Journal of Arts, Literature, Humanities and Social Sciences

www.jalhss.com

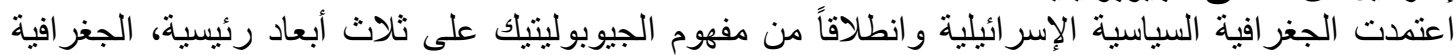

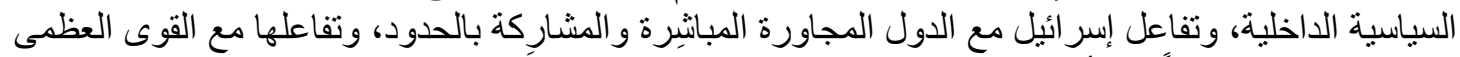

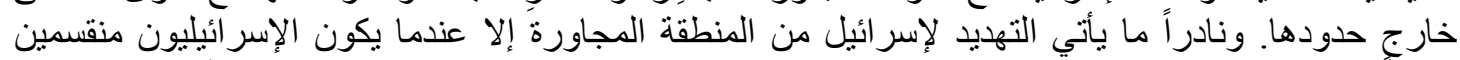

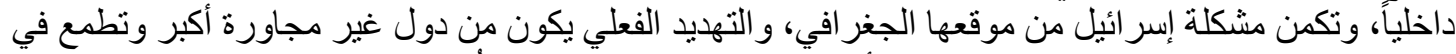

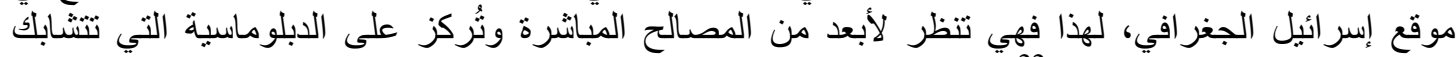

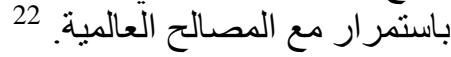

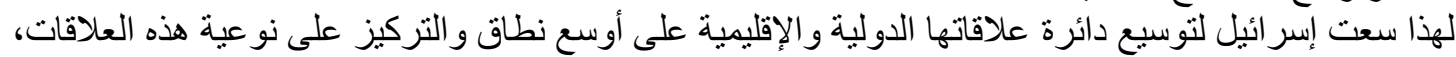

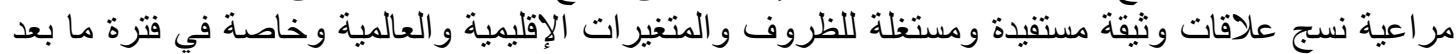

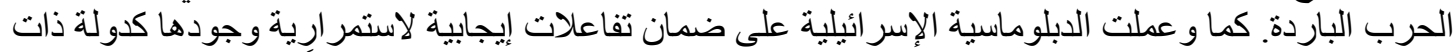

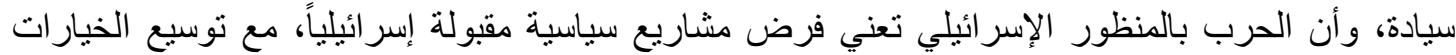

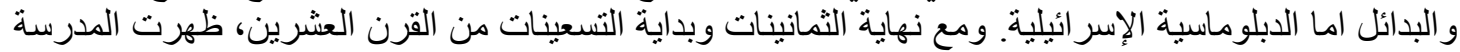

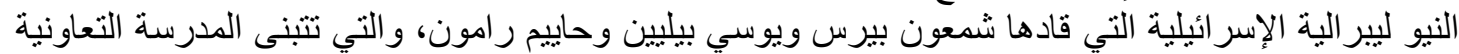

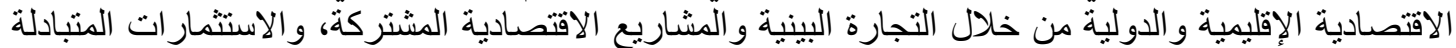

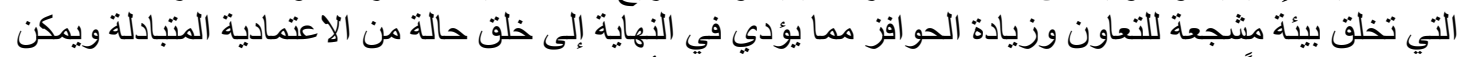

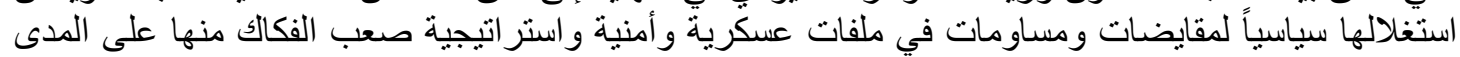

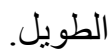
هذه المدرسة عملت على إحداث و عي طبقي يتخطى الحدود القومية والوطنية لدى النخب العربية و الفلسطينية

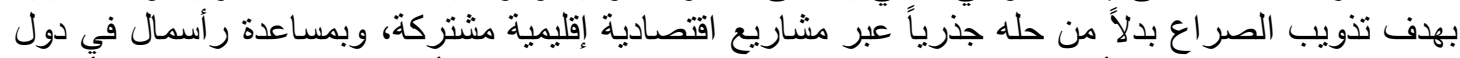

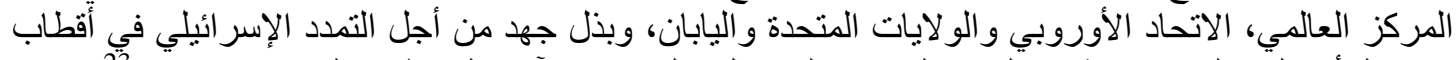

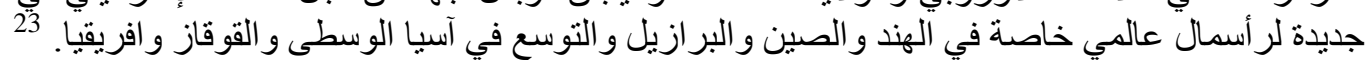

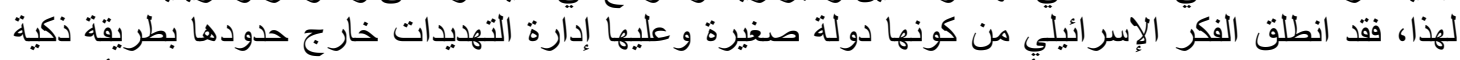

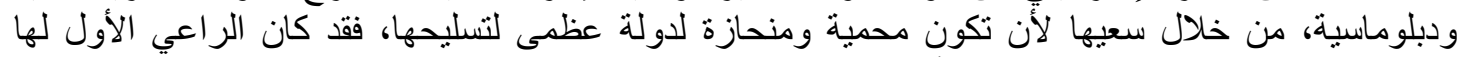

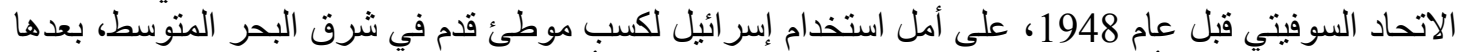

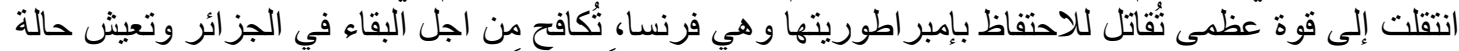

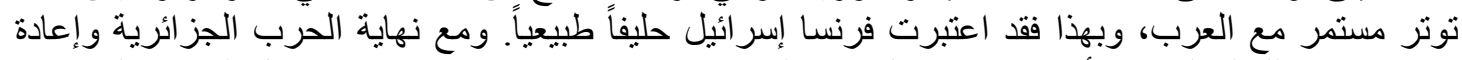

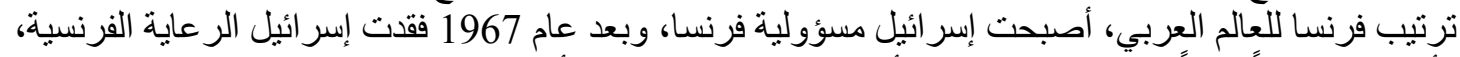

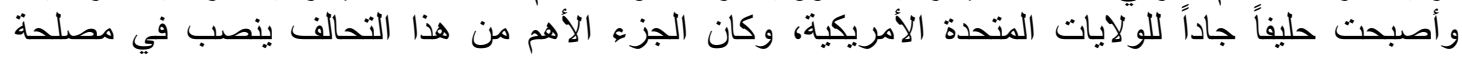

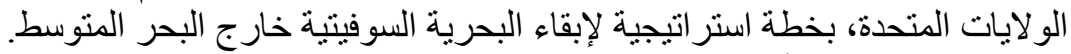

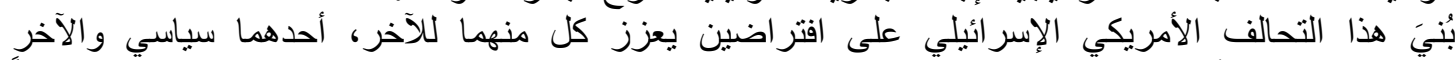

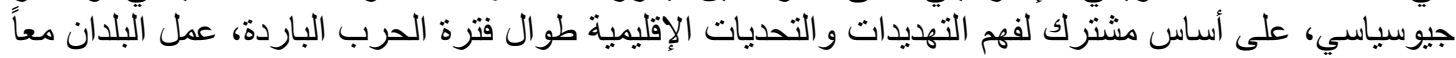

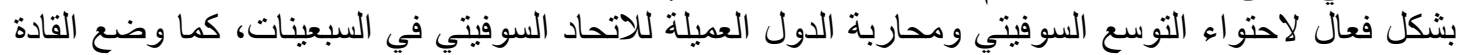

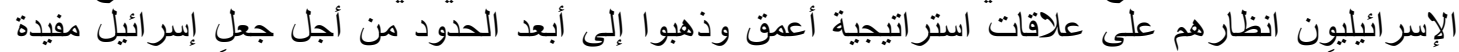

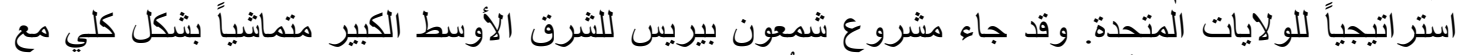

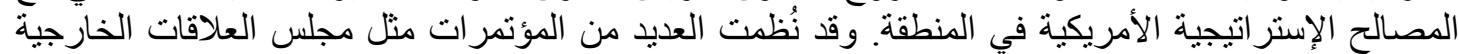

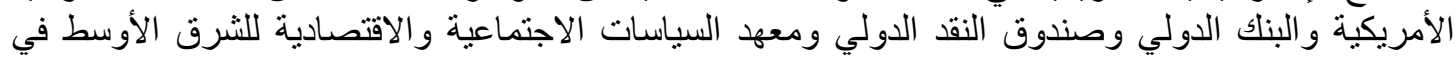

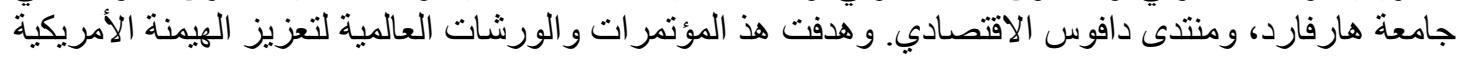

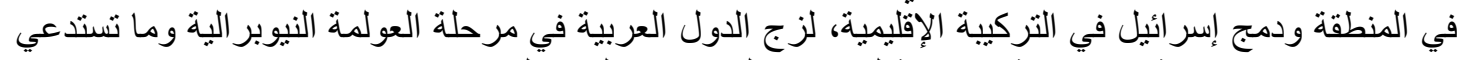
من إصلاحات اقتصادية واجتماعية وسياسية لتنتق مع المقتر ح الإقليمي الجديد.

22 -The Geopolitics of Israel: Biblical and modern, STRATFORD

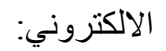
الموقع http://cdn2.hubspot.net/hubfs/515194/B2C_content/B2CWF3/Geopolitcs_of_Israel_ebook.pdf?t=1443541217488

23- شمعون بيريس، الثرق الأوسط الجديد (ترجمة محمد حلمي حافظ)، عمان: الأهلية للنشر،1998 


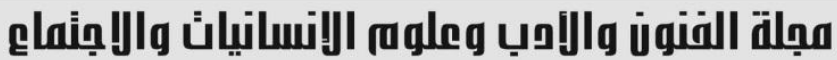

Journal of Arts, Literature, Humanities and Social Sciences www.jalhss.com

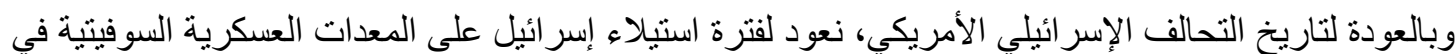

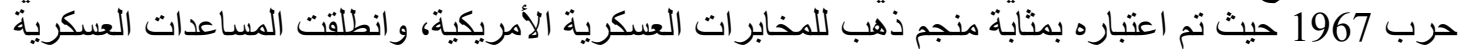

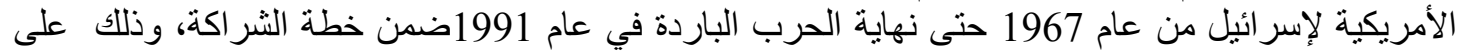

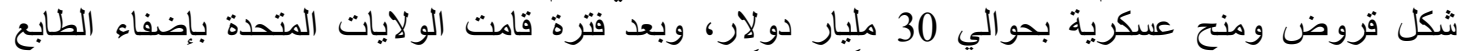

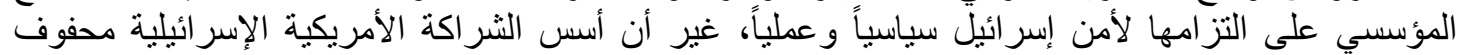

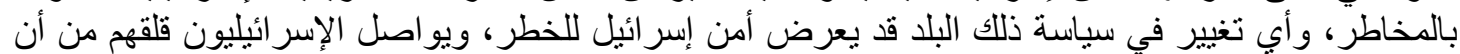

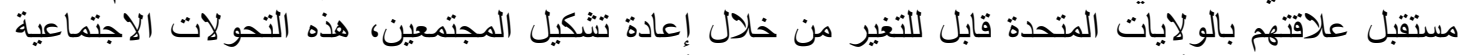

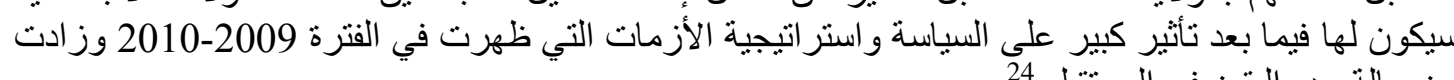
من حالة عدم اليقين في المستقبل. 24 هذا، التخوف والقلق دفع بالفكر الإسر ائيلي الجيوبوليتيكي للبحث عن منافذ أخرى وتحالفات جديدة تخدم وتؤمن

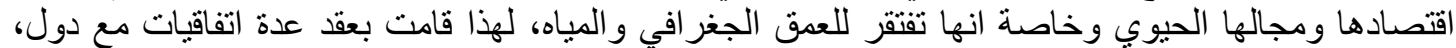

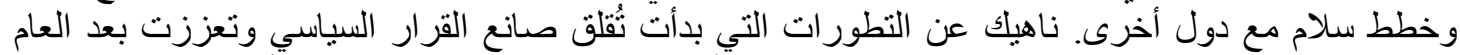

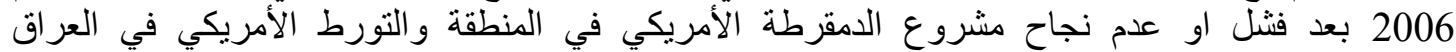

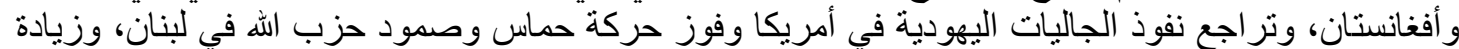
تأثير موجات المد الإسلامي كل هذه العو امل وغير ها دفعت بإسر ائيل إلى إعادة التفكير في توسيع دائرة تحالفاتها وخيار اتها الإقليمية. من هنا، ستحاول الدراسة الوقوفة على الاتفاقيات الإسرائيلية مع بعض الدول وذلك محاولة من إسرائيل

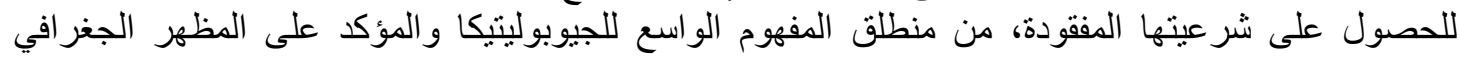

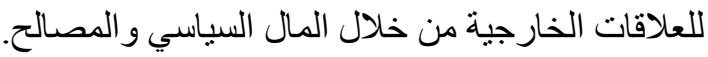

أولاً: إسر ائيل ودول إفريقيا

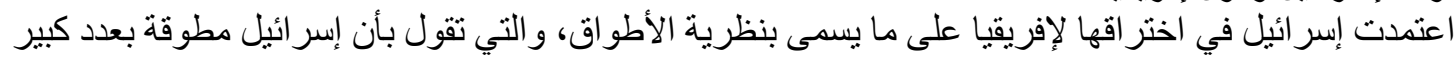

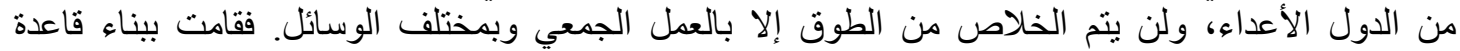

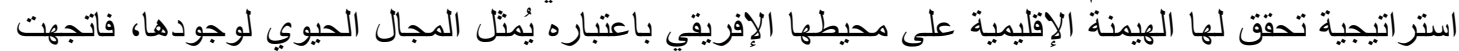

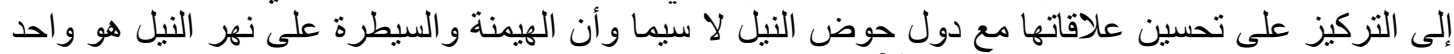
من الأهداف الحيوية والتاريخية لإسر ائيل. 26

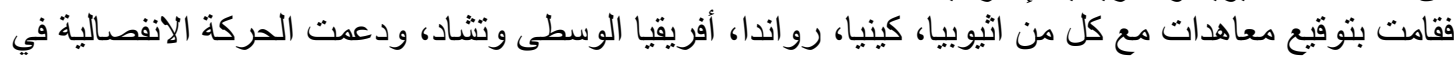

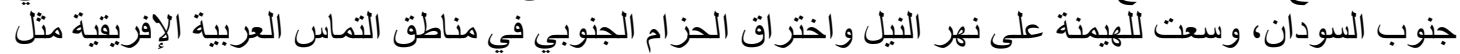

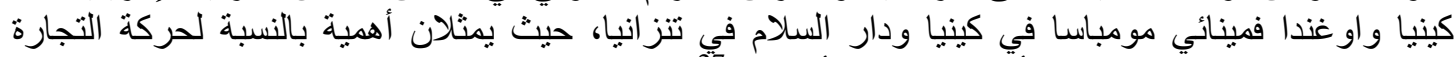

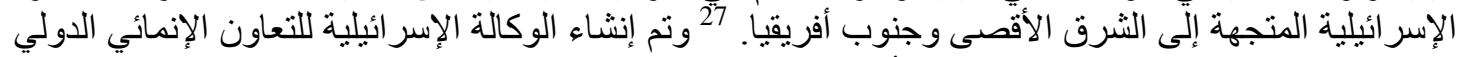

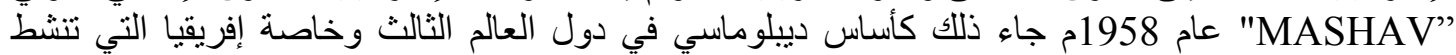

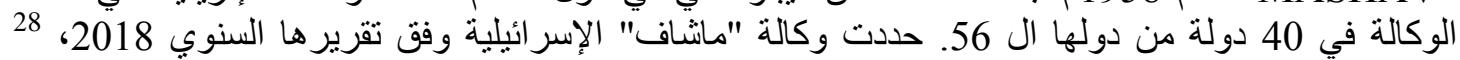

24 -THE FUTURE OF THE U.S.- ISRAEL STRATEGIC PARTNERSHIP, HAIM. MALKA, CSIS (CENTER FOR STRATEGIC \& INTERNATIONAL STUDIES

إنظر الكتروني: public/legacy_files/files/publication/110908_Malka_CrossroadsUSIsrael_Web.pdf

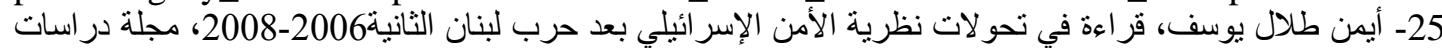

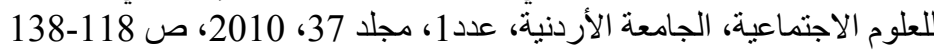

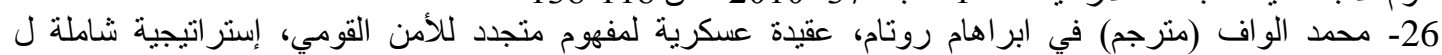

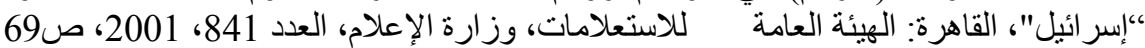

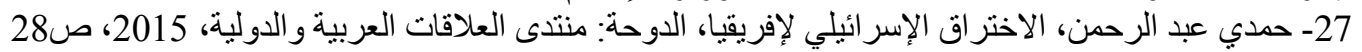
28 -The annual report of Israel's agency for international cooperation "MASHAV", 2018, p 20.

https://mfa.gov.il/MFA/mashav/Publications/Annual_Reports/Documents/AnnualReport2018. pdf 


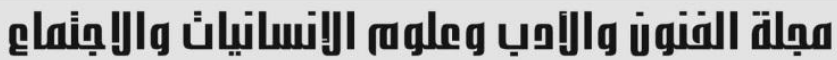

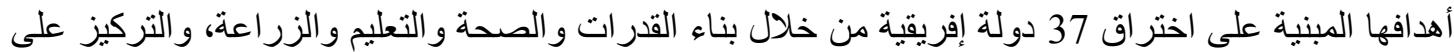

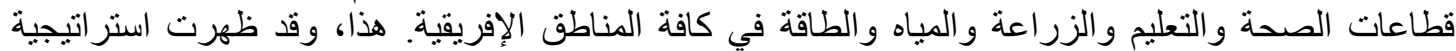

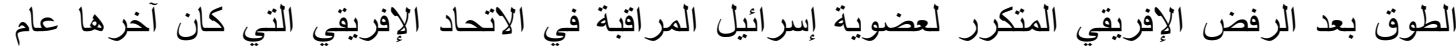

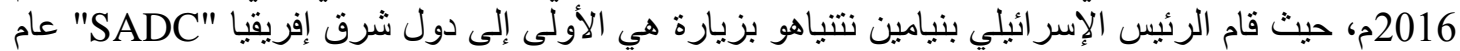

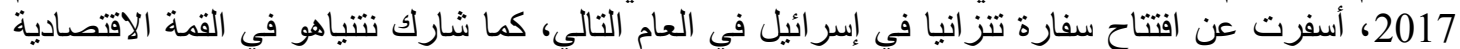

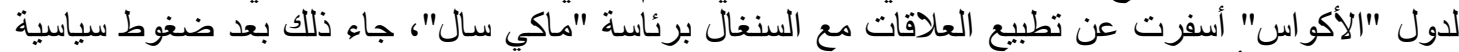

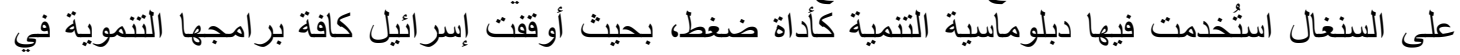

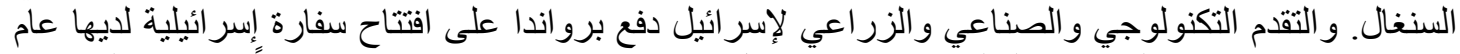

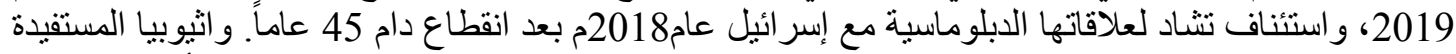

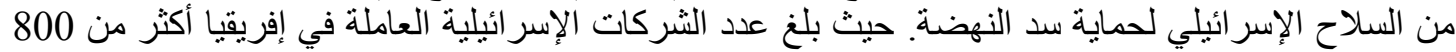

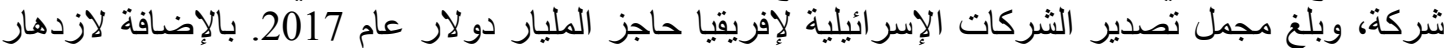

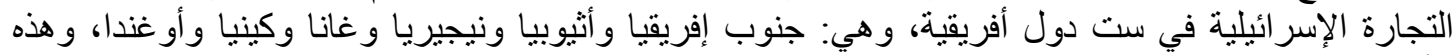

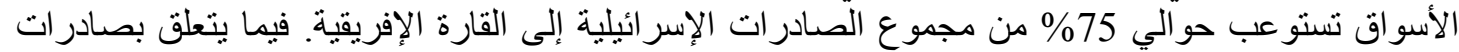

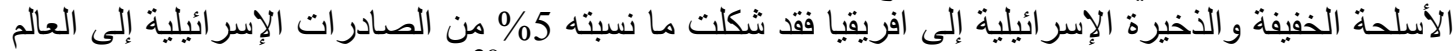

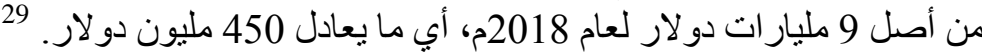

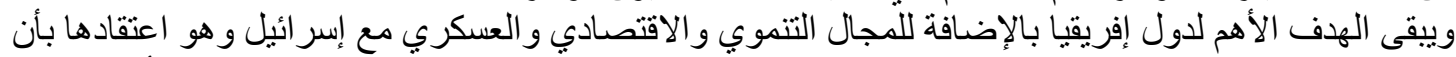

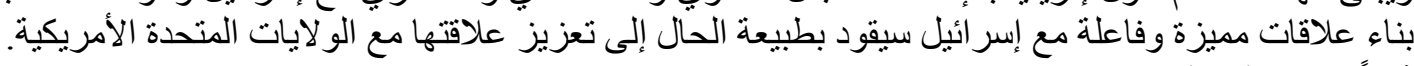

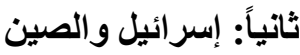

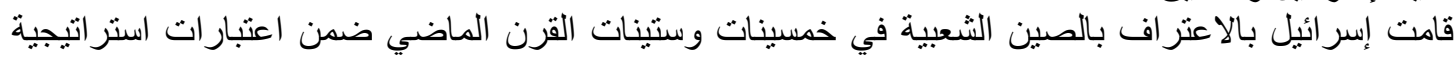

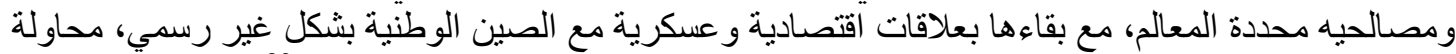

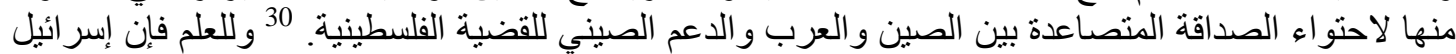

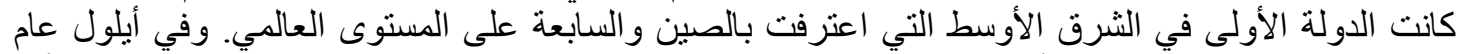

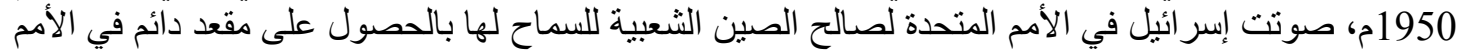

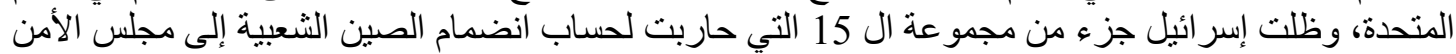
كعضو دائم. لقد بدأت مأنسة العلاقات الثنائية الصينية الإسر ائبلية الرسمية في شهر كانون الثاني 1992م، أي بعد أسابيع من

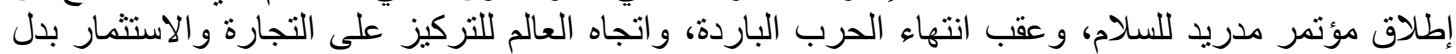

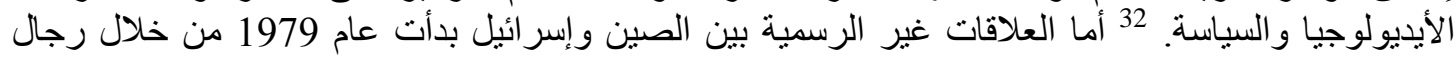

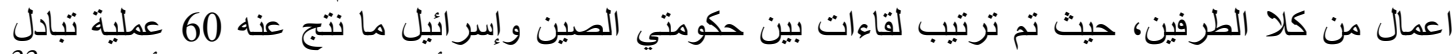

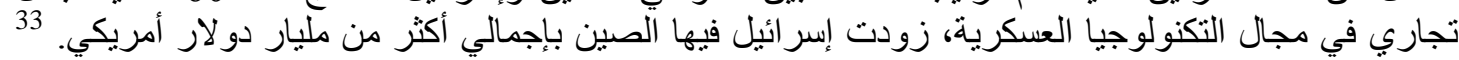

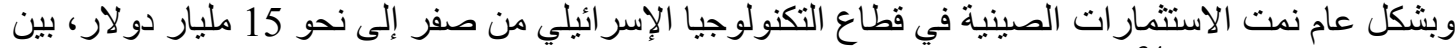

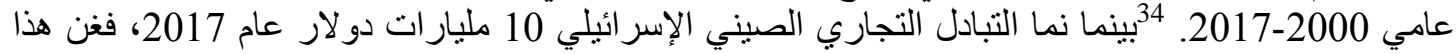

29 - وكالة عرب 48، سعي إسرائيل للتغلغل في أفريقيا عبر منافذ تجارية، https://www.arab48.co 2019/12/9

30 -Yitzhak Schichor,Israel's Military Transfers to China and Tawian,Survival ، no, 1 ,Vol.40.1998, p.68-90

31- عبد العزيز حمدي عبد العزيز، العلاقات الصينيةـ الإسر ائلية، السياسة الدولية العدد132، ابريل 2998، ص.127-

32 -Pevzner, Alexender B., A New Era for Israel- China Relations, Jerusalem Post, 23 January 2018

33 -Efron, Shira, Howard J. Shatz, Arthur Chan, Emily Haskel, Lyle J. Morris, and Andrew Scobell, The Evolving Israel-China Relationship, (California: Rand Corporation 2019)، p.xii 34 -Pevzner, Alexender B., A New Era for Israel- China Relations, Jerusalem Post, 23 Januaryy 2018 
المبلغ نما حو الي 30\% عام 2018، رغم الحرب التجارية الصينية الأمريكية، وتُعتبر الصين أكبر شريك تجاري

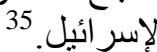

كما وشهدت الأشهر الستة الأولى من العام 2019، سلسلة صفقات كبيرة، اشترت فيها الصين حصصاً من لئن

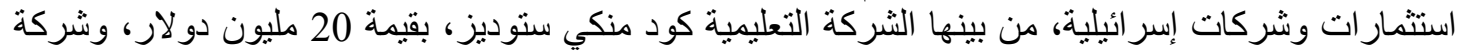

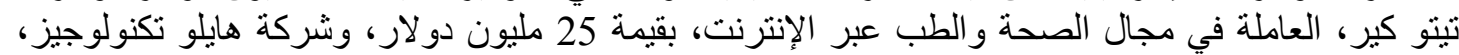

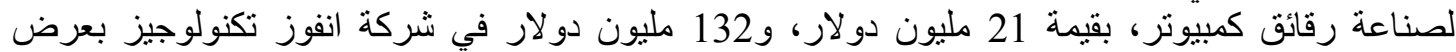

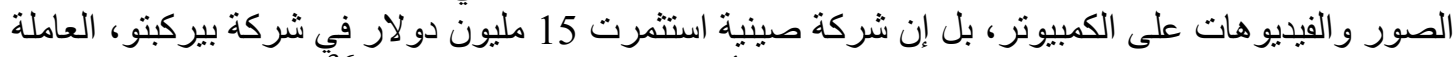

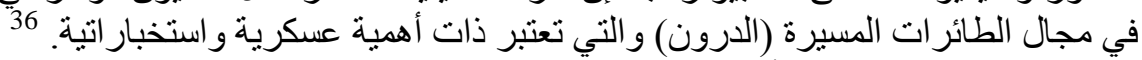

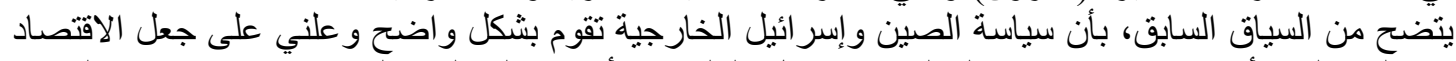

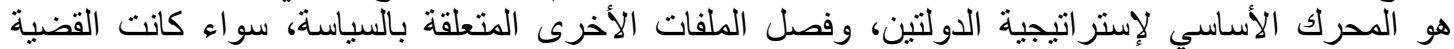

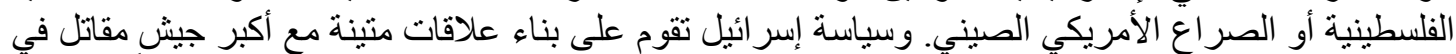

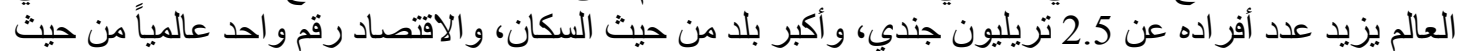

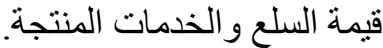

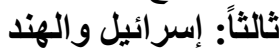

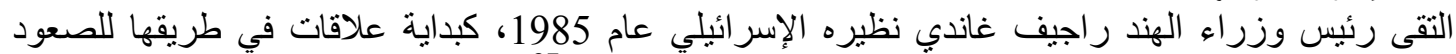

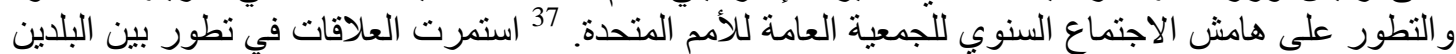

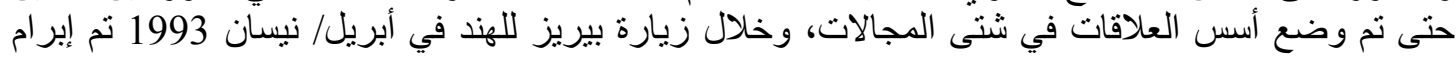

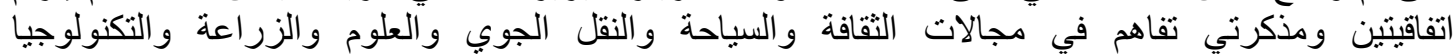

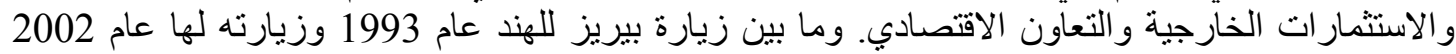

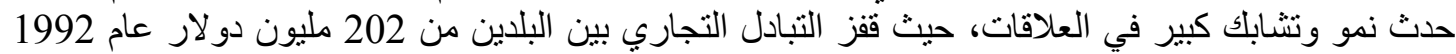

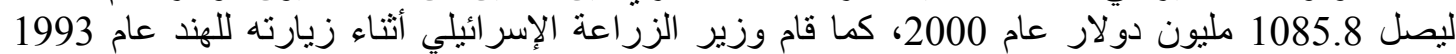

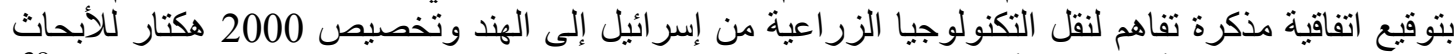

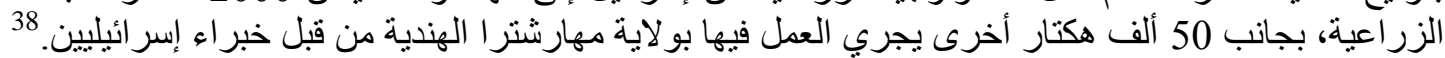

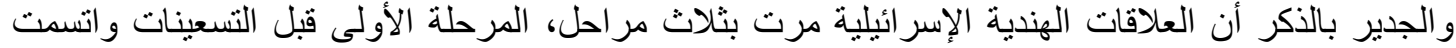

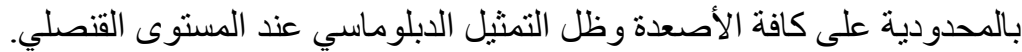

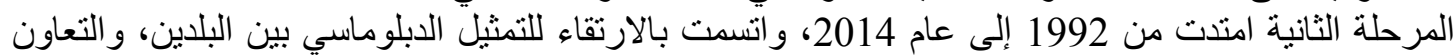

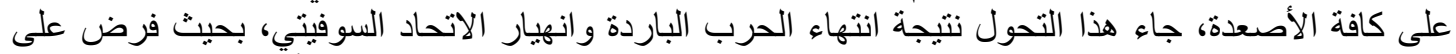

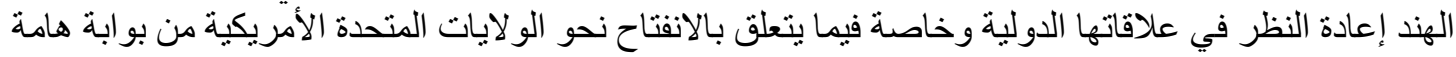

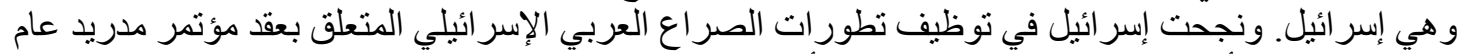

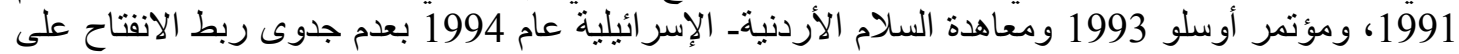
إسر ائيل بمسار الصر اعزع العربي الإسر ائيلي.

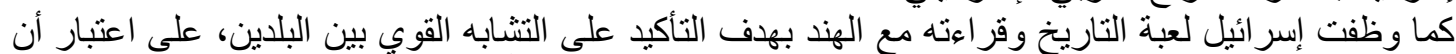

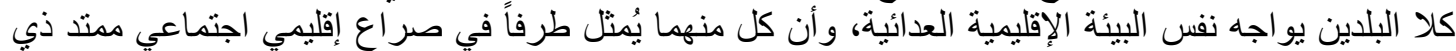

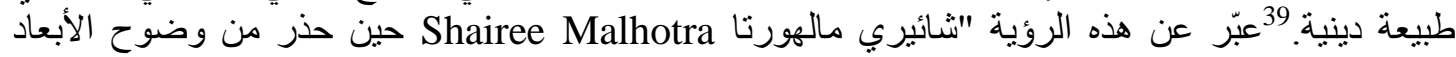

35 -Lazaroof, Tovah, Netanyahu pushes for free trade with China in 2019, Jerusalem Post, 25 October 2018

36 -Barak, Dorian and Yuelin Dang, Israel navigates US-China trade tensions, Asia Times,

29 June 2019

37 -India and Israel's Secret Love Affair: Jayita Sarkar, The Nations' Interact 10/12/2014 https://goo.gl/tDnpi8

38- مستقبل التحالف الهندي الإسرائيلي: مدحت أيوب، موقع الجزيرة، 3/10/2004، تاريخ المشاهدة 7/7/2017، https://goo.gl/Xq6M89 الريطي 39- 3ي لمزيد من التفصيل:

Wald, Shalom Salomon and Arielle Kandel, INDIA, ISRAEL, AND THE JEWISH PEOPLE Looking Ahead, Looking Back 25 Years after Normalization (Jerusalem: The Jewish People Policy Institute, 2017), 
الدينية في علاقات البلدين و أنه ليس من مصلحة إسر ائيل تجذر هذا البعد الديني. 40 وساعدت البيئة الصر اعية في

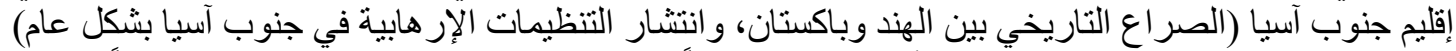

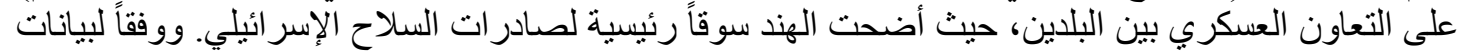

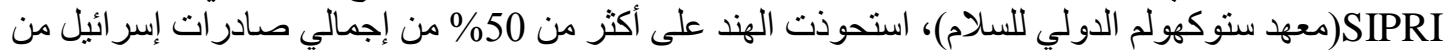

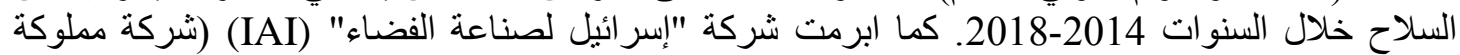

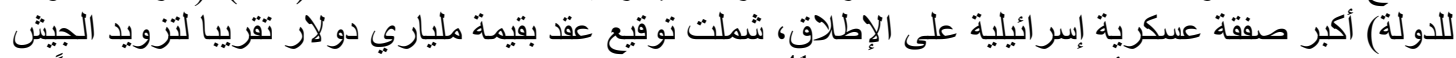

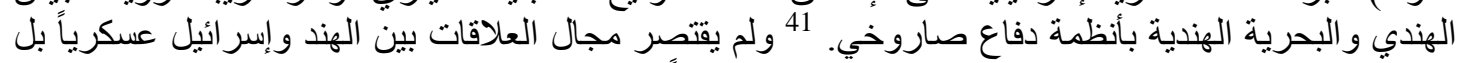

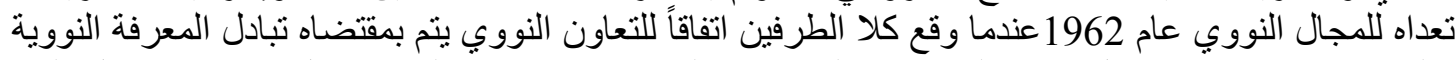

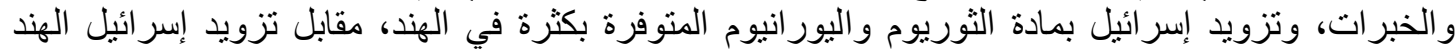
بالتكنولوجيا النووية الجديدة.

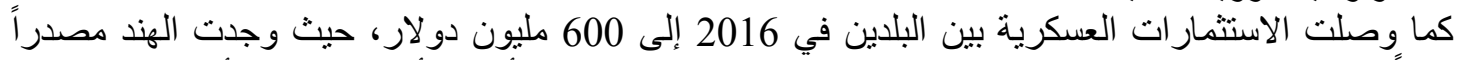

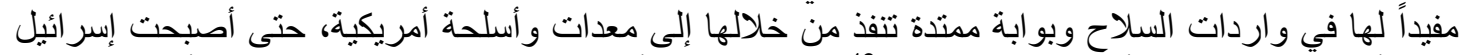

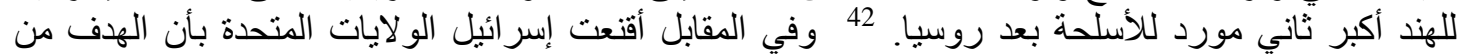

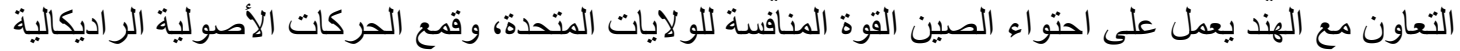

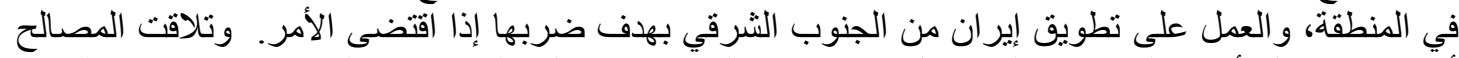

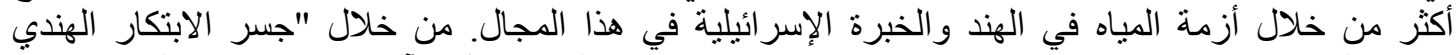

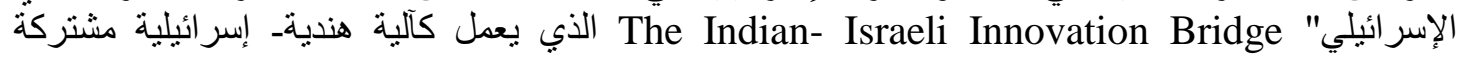

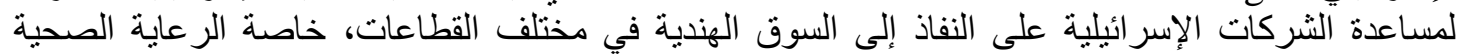

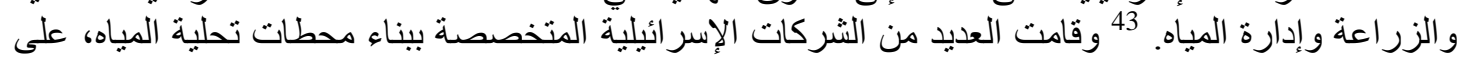

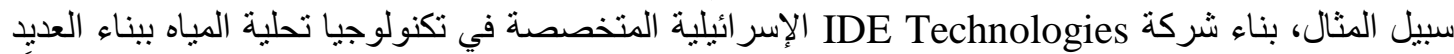

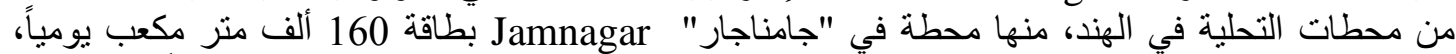

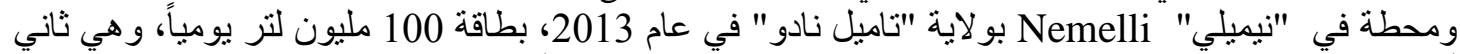

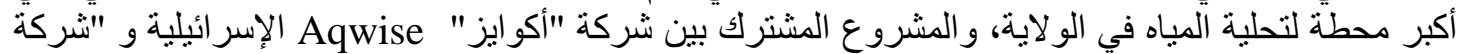

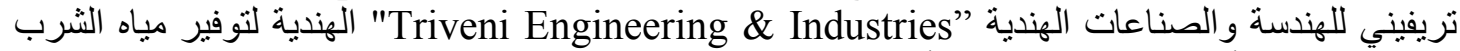

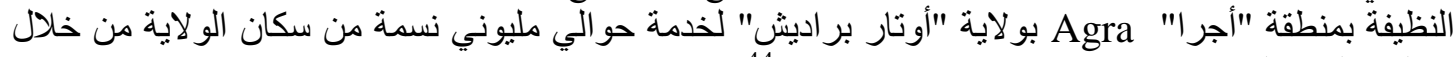

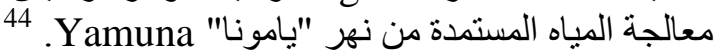

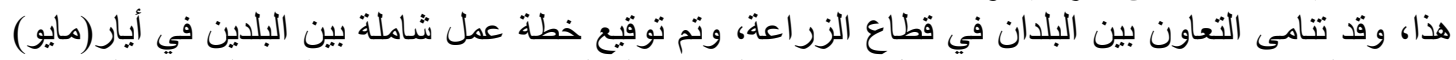
2006، لتنفيذ مشروعات ثنائية من خلال "مركز التعاون التعاون الدولي" MASHAV التابع لوزارة الخارين الخارجية

http://Bit.ly/2XNFDcQ (accessed on October 2, 2019).

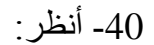

"The India-Israel relationship is not ideological, but it is being framed as such: Shairee Malhotra", Interview by Praveen Donthi, The Caravan, 14 March 2019. Available at: http://bit.ly/37ChdHO (accessed on September 10, 2019).

41 -Stockholm International Peace Research Institute, SIPRI Arms Transfers Database, Available at: https://www.sipri.org/databases/armstransfers (accessed on September 20, 2019)

42 -Israeli defense minister lands at India airshow to boost arms sales: Tommy Wilkes, Reuters, $18 / 2 / 2015$, https://goo.gl/oNv7FS

$$
\text { 43- لكزيد من التفصيل حول هذه الآلية: }
$$

Bajaj, Kashika, "Meet the Six Startups from India-Israel Innovation Bridge Challenge", Inc 42, 03 Aug. 2018. Available at: http://bit.ly/2Dh3gRR (accessed on October 2, 2019) 44- لكزيد من التفصيلات حول العقود و المشروعات التي تنفذها الثركات الإسر ائيلية داخل قطاع المياه والزراعة في .

"Bilateral Trade Relations", Embassy of India in Israel website. Available at:

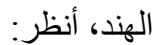
https://www.indembassyisrael.gov.in/pages?id=nel5a\&subid=vbmOe 


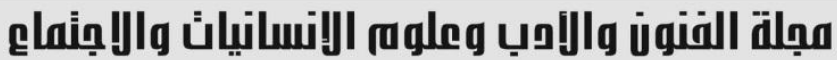

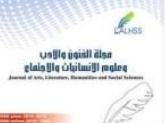

Journal of Arts, Literature, Humanities and Social Sciences

www.jalhss.com

Volume (63) January 2021

2021 يناير

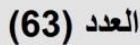

الإسرائيلية، و "مركز التعاون الدولي" CINADCO التابع لوزارة الزراعة والتتمية الريفية الإسرائيلية،

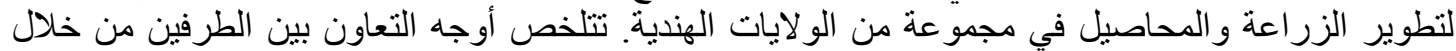

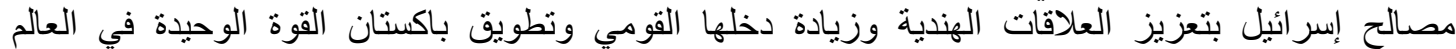

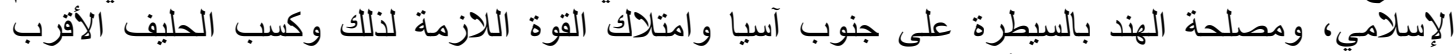
لإسر ائيل و هي الو لايات المتحدة الأمريكية.

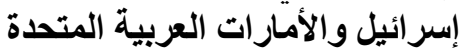

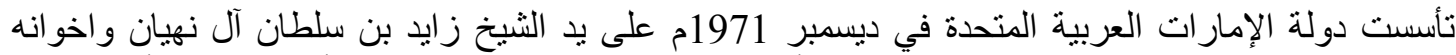

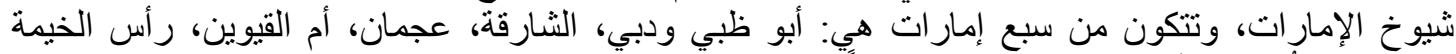

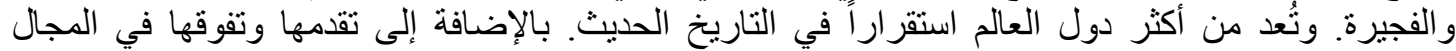
الاقتصادي و الاجتماعي و الأمن. الإن. اقتصادياً، بلغت الإمارات العربية المتحدة الأعلى على مستوى دول الخليج، حيث وصلت استثمار اتها الخارجية

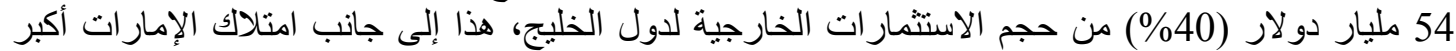

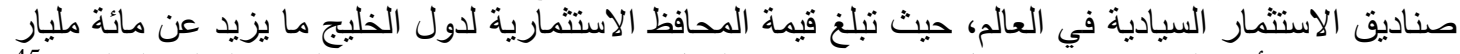

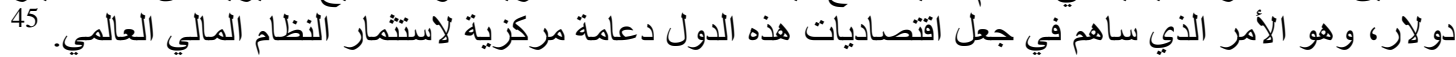

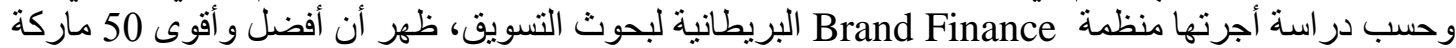

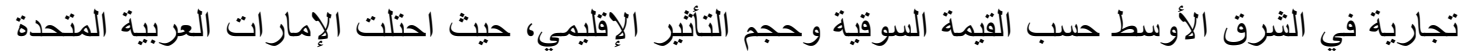

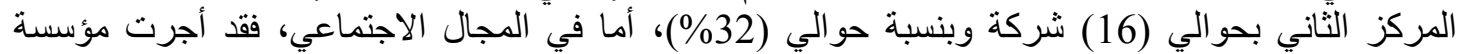

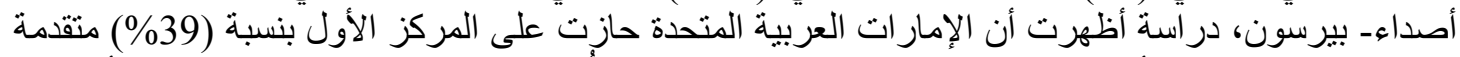

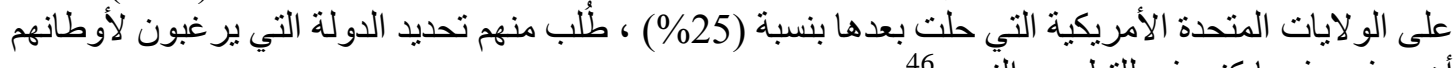

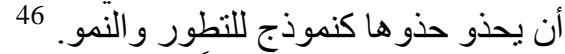

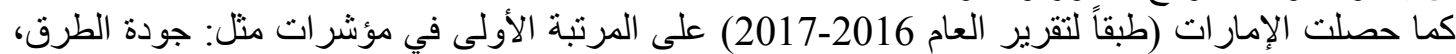

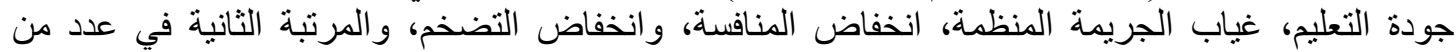

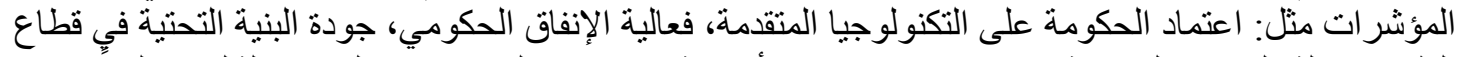

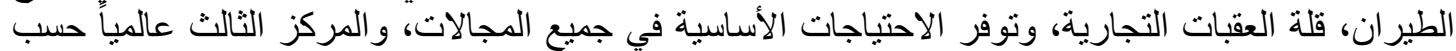

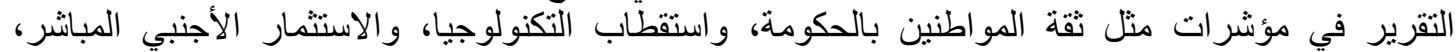

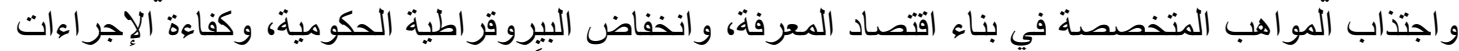

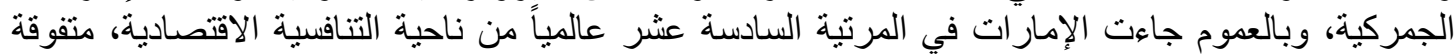

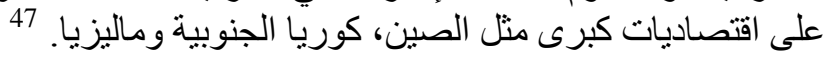

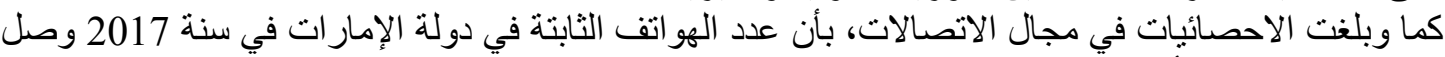

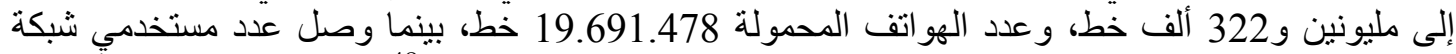

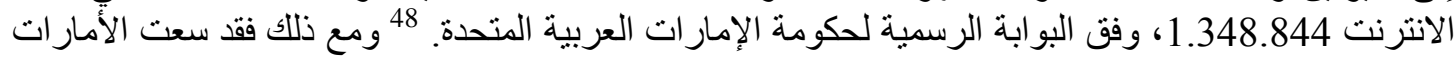

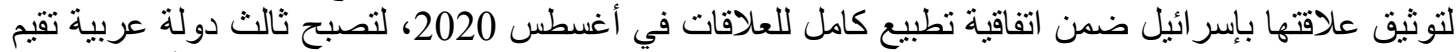

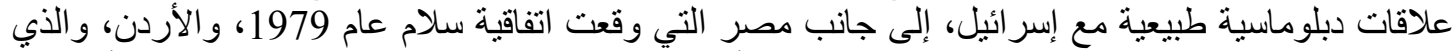

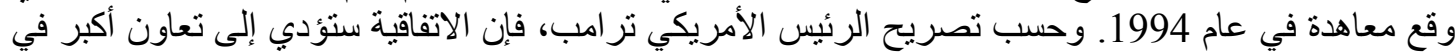
السياحة و الأمن و الطاقة و التكنولوجيا و وجالات أخرى عديدة.

45- الأمانة العامة لهجلس التعاون الخليجي، إدارة البحوث و الدراسات (2010)، مقومات وفرص الاستثمار في دول

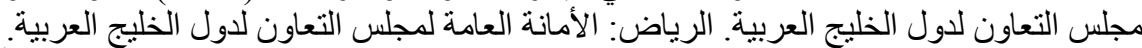

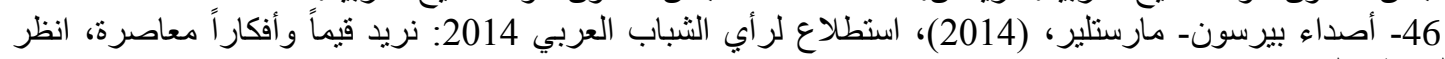

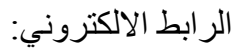

http://arabyouthsurvey.com/wp-content/themes/arabyouth-english/downloads/AYS-

Whitepaper-ar.pdf

47 -Economic Forum -Schwab, K. ed. (2016): The Global Competitiveness Report 20162017. Geneva: The World

48- عبد الله على طابور، تطور المؤسسات الإعلامية في دولة الإمارات وأثرها في التتمية الثقافية، أبو ظبي: المجمع الثقافي،2000 


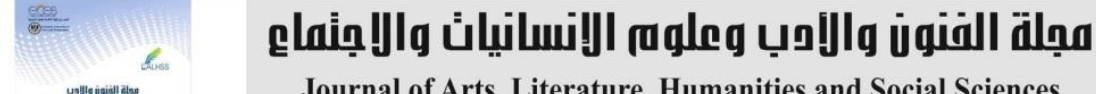 www.jalhss.com}

على الرغم من محافظة الإمارات وإسر ائيل على العلاقات غير المعلنة قبل توقيع الاتفاق، من خلال استضافت

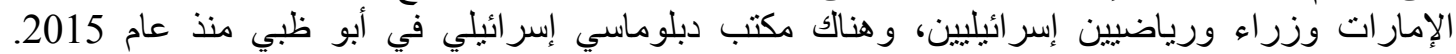

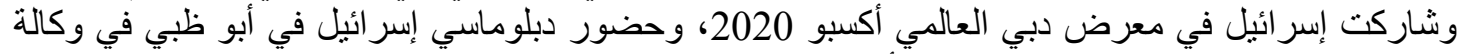

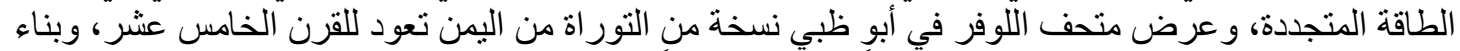

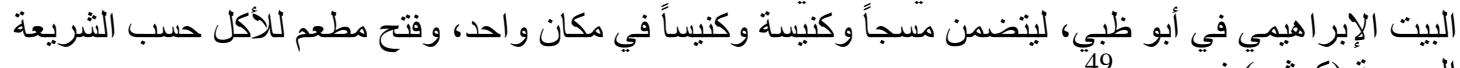
اليهودية (كوشر) في دبي.

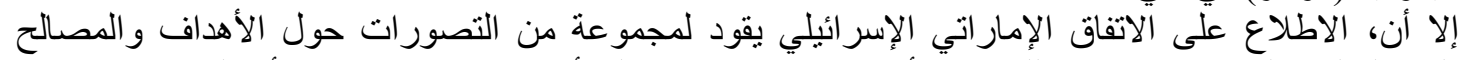

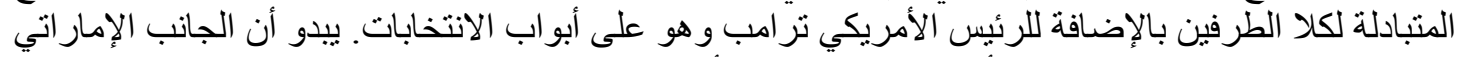

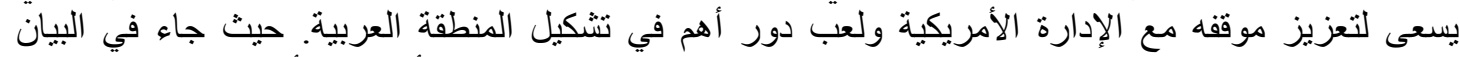

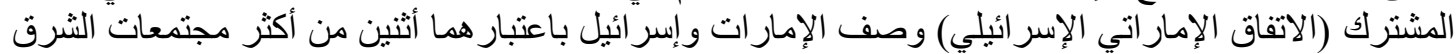

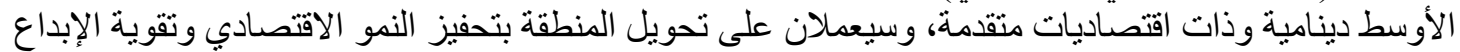

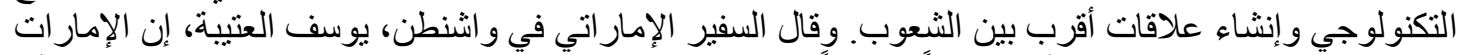

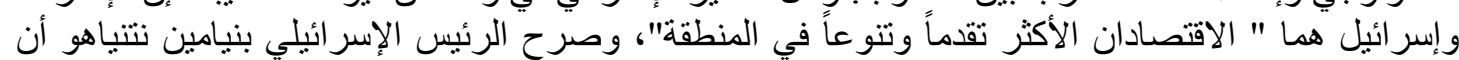

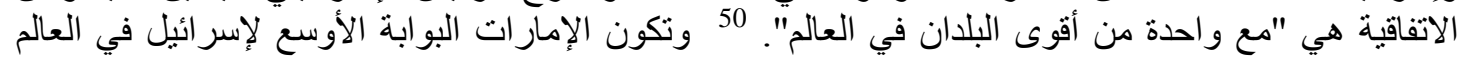
العربي. كما استطاعت إسر ائيل ضم الإمارات لعلاقاتها الجيوبوليتيكية، وبذلك تفتح الطريق أمام باقي الدول العربية لتقبل

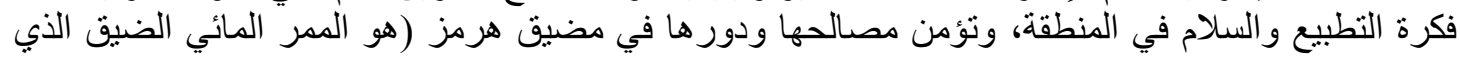

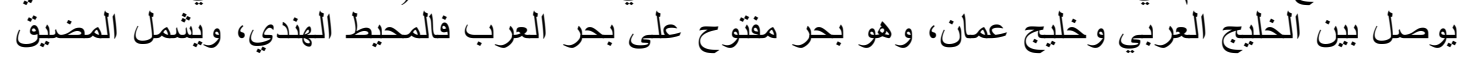

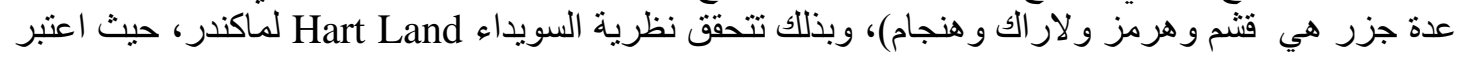

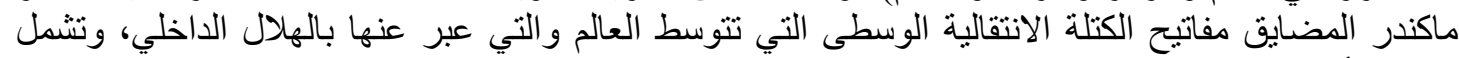

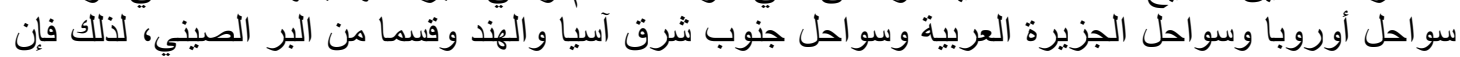

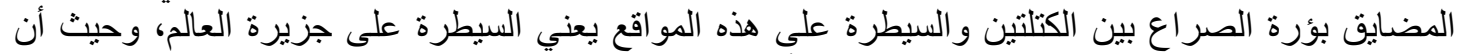

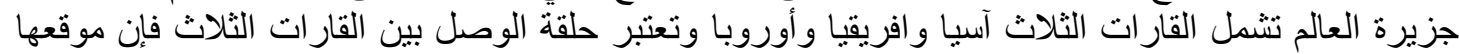

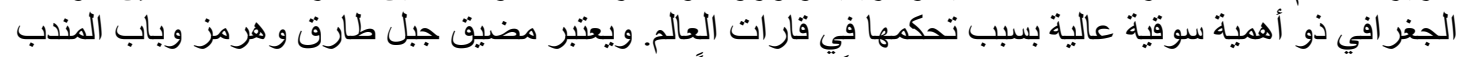

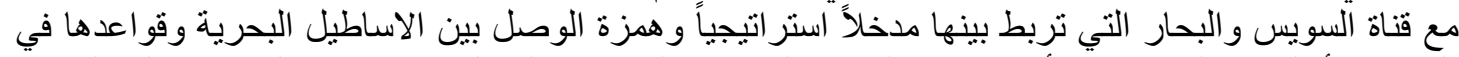

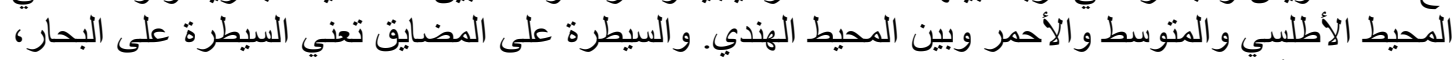

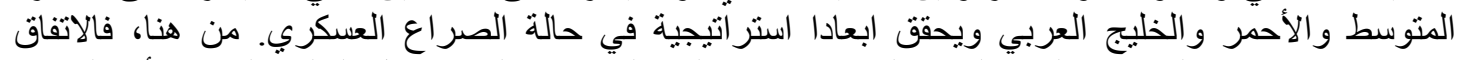

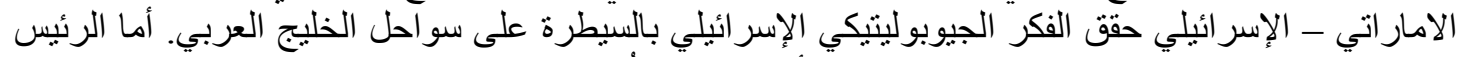

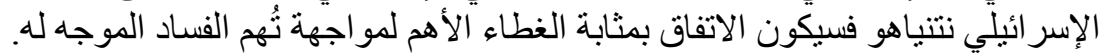

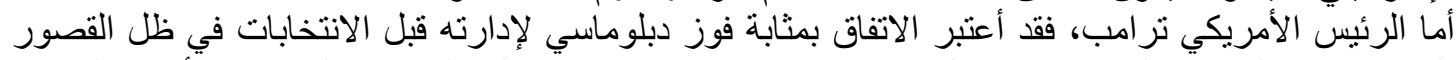

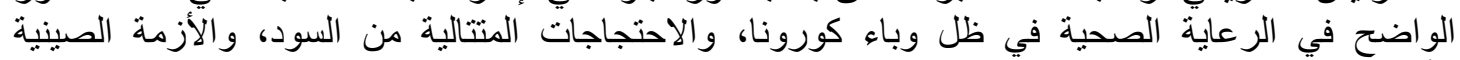
الأمريكية.

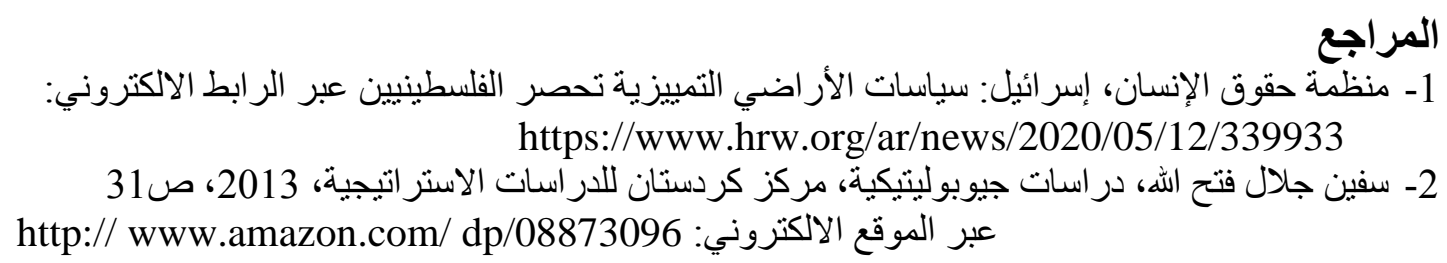
- 3Kenichi Ohama, "Managing in borderless world", Harvard business review,5$6 / 1989$

49 -Yousef al Otaiba, Annexation will be a serious setback for better relations with the Arab world, Ynet, 12 June 2020 50 - Lahav Harakov, Israel, UAE Reach Historic Peace Deal: 'We can Make a Wonderful Future'، The Jerusalem Post, 14 August 2020 


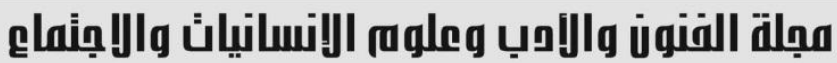

Journal of Arts, Literature, Humanities and Social Sciences www.jalhss.com

4- محمد رياض، الأصول العامة في الجغر افيا السياسية والجيوبوليتيك، (2012)، مؤسسة هنداوي للتعليم

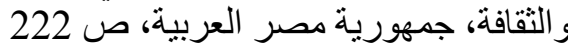

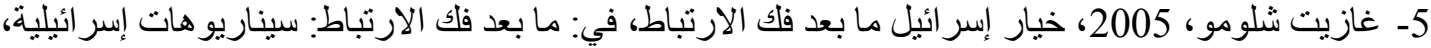

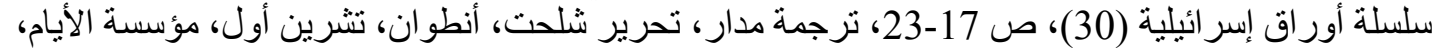
رام الله، فلسطين

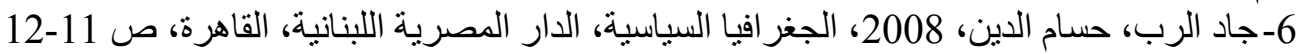

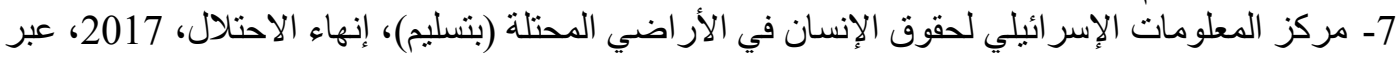

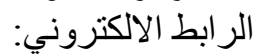

https://www.btselem.org/arabic/duty_to_end_occupation

-8 McClory,J.2015, The Soft Power 30: Global Ranking of Soft Power. Portland Communications [online] Available at: www.portlandcommunication.com/downloads/the- soft-power-3020\% Nov.pdf [Accesses Dec30.2015]

N. Pounds, Political Geography, McGraw Hill 1963, p.407 -9

-10 Ancel,Les Frontieres, Etudc de Geographic Politique, Recueil de Couurs, pp. 207-297.1936

$$
\text { أنظر أيضاً: محمد رياض، الأصول العامة في الجغر افيا السياسية مرجع سابق، ص199 : }
$$

-11 The Geopolitics of Israel: Biblical and modern, STRATFORD

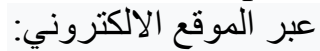
http://cdn2.hubspot.net/hubfs/515194/B2C_content/B2CWF3/Geopolitcs_of_Israel_-

ebook.pdf?t=1443541217488

12- شمعون بيريس، الثرق الأوسط الجديد (ترجمة محمد حلمي حافظ)، عمان: الأهلية للنشر،1998

THE FUTURE OF THE U.S.- ISRAEL STRATEGIC PARTNERSHIP, HAIM. MALKA, CSIS (CENTER FOR STRATEGIC \& - INTERNATIONAL STUDIES

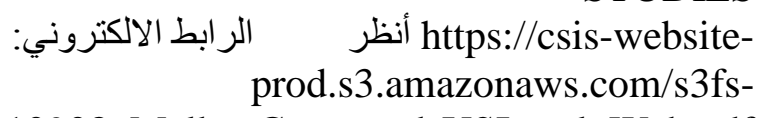

public/legacy_files/files/publication/110908_Malka_CrossroadsUSIsrael_Web.pdf 14- أيمن طلال يوسف، قراءة في تحولات نظرية الأمن الإسر ائيلي بعد حرب لبنان الثانية_2006-2008، مجلة در اسات للعلوم الاجنماعية،

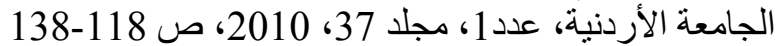

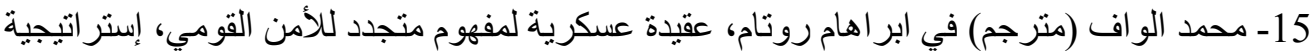

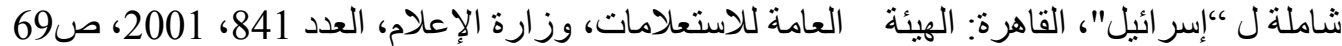
16- حمدي عبد الرحمن، الاختراق الإسر ائيلي لإفريقيا، الدوحة: منتدى العلاقات العراتية العربية و الدولية، 2015،

1 -The annual report of Israel's agency for international cooperation "MASHAV", 2018, p 20.

https://mfa.gov.il/MFA/mashav/Publications/Annual_Reports/Documents/AnnualRep ort2018.pdf 17- وكالة عرب 48، سعي إسر ائيل للتغلغل في أفريقيا عبر منافذ تجارية، https://www.arab48.co 2019/12/9 بتاريخ -18 Yitzhak Schichor,Israel's Military Transfers to China and Tawian,Survival ، no, 1,Vol.40.1998, p.68-90

19- عبد العزيز حمدي عبد العزيز، العلاقات الصينيةـ الإسر ائيلية، السياسة الدولية العدد132، ابريل 


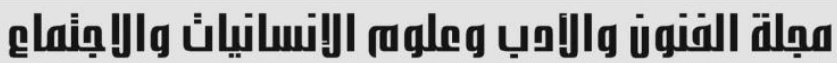 \\ Journal of Arts, Literature, Humanities and Social Sciences www.jalhss.com}

Volume (63) January 2021

العدد (63) يناير 2021

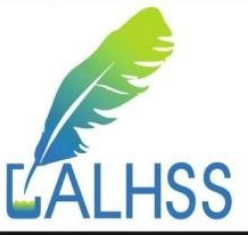

-20 Pevzner, Alexender B., A New Era for Israel- China Relations, Jerusalem Post, 23 January 2018

-21 Efron, Shira, Howard J. Shatz, Arthur Chan, Emily Haskel, Lyle J. Morris, and Andrew Scobell, The Evolving Israel-China Relationship, (California: Rand Corporation 2019)، p.xii

-22 Pevzner, Alexender B., A New Era for Israel- China Relations, Jerusalem Post, 23 Januaryy 2018

-23 Lazaroof, Tovah, Netanyahu pushes for free trade with China in 2019, Jerusalem Post, 25 October 2018

-25 Barak, Dorian and Yuelin Dang, Israel navigates US-China trade tensions, Asia Times, 29 June 2019

-26 India and Israel's Secret Love Affair: Jayita Sarkar, The Nations' Interact 10/12/2014 https://goo.gl/tDnpi8

$$
\begin{aligned}
& \text { 27- مستقبل التحالف الهندي الإسر ائبلي: مدحت أيوب، موقع الجزيرة، 3/10/2004، تاريخ المشاهدة } \\
& \text { 7/7/2017 الر ابط: } \\
& \text { https://goo.gl/Xq6M89 }
\end{aligned}
$$

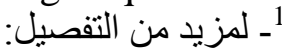

Wald, Shalom Salomon and Arielle Kandel, INDIA, ISRAEL, AND THE JEWISH PEOPLE Looking Ahead, Looking Back 25 Years after Normalization (Jerusalem: The Jewish People Policy Institute, 2017),

$$
\text { http://Bit.ly/2XNFDcQ (accessed on October 2, 2019). }
$$

"The India-Israel relationship is not ideological, but it is being framed as such: Shairee Malhotra", Interview by Praveen Donthi, The Caravan, 14 March 2019. Available at: http://bit.ly/37ChdHO (accessed on September 10, 2019)

-29 Stockholm International Peace Research Institute, SIPRI Arms Transfers Database, Available at: https://www.sipri.org/databases/armstransfers (accessed on September 20, 2019)

-30 Israeli defense minister lands at India airshow to boost arms sales: Tommy Wilkes, Reuters, 18/2/2015, https://goo.gl/oNv7FS

$$
\text { 31 - لمزيد من التفصيل حول هذه الآلية: }
$$

Bajaj, Kashika, "Meet the Six Startups from India-Israel Innovation Bridge Challenge", Inc 42, 03 Aug. 2018. Available at: http://bit.ly/2Dh3gRR (accessed on October 2, 2019)

$$
\text { و الزر اعة في المزند، أنظر: التفيلات حول العقود و المشروعات التي تتفذها الثركات الإسر ائيلية داخل قطاع المياه }
$$

"Bilateral Trade Relations", Embassy of India in Israel website. Available at:

https://www.indembassyisrael.gov.in/pages?id=nel5a\&subid=vbmOe

33- الأمانة العامة لمجلس التعاون الخليجي، إدارة البحوث و الدر اسات (2010)، مقومات وفرص

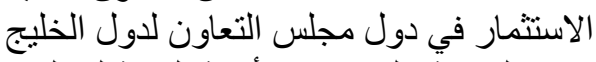

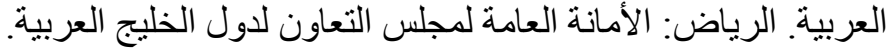

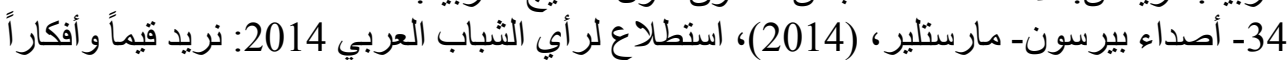
معاصرة، انظر الر ابط الالكتروني:

http://arabyouthsurvey.com/wp-content/themes/arabyouth-

english/downloads/AYS-Whitepaper-ar.pdf 


\section{مِالة القَو}

Journal of Arts, Literature, Humanities and Social Sciences

www.jalhss.com

Volume (63) January 2021

العدد (63) يناير 2021

-35 Economic Forum -Schwab, K. ed. (2016): The Global Competitiveness

Report 2016-2017. Geneva: The World

36- عبد الله على طابور، تطور المؤسسات الإعلامية في دولة الإمارات و أثر ها في التنمية الثقافية، أبو

ظبي: المجمع الثقافي،2000

-37 Yousef al Otaiba, Annexation will be a serious setback for better relations with the Arab world, Ynet, 12 June 2020

-38 Lahav Harakov, Israel, UAE Reach Historic Peace Deal: 'We can Make a

Wonderful Future'، The JerusalemPost, 14 August 2020 